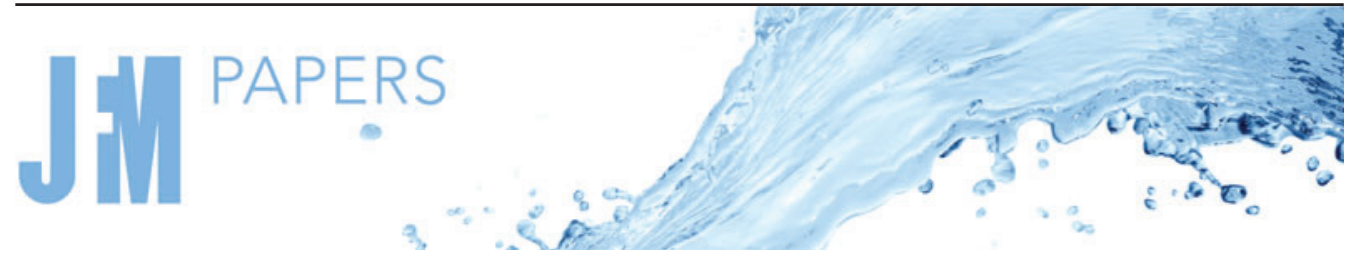

\title{
A macroscopic two-length-scale model for natural convection in porous media driven by a species-concentration gradient
}

\author{
Stefan Gasow ${ }^{1}$, Andrey V. Kuznetsov ${ }^{2}$, Marc Avila ${ }^{1,3}$ and Yan Jin ${ }^{1,3, \dagger}$ \\ ${ }^{1}$ Center of Applied Space Technology and Microgravity (ZARM), University of Bremen, 28359 Bremen, \\ Germany \\ ${ }^{2}$ Department of Mechanical and Aerospace Engineering, North Carolina State University, Raleigh, NC \\ 27695-7910, USA \\ ${ }^{3}$ MAPEX Center for Materials and Processes, University of Bremen, 28359 Bremen, Germany
}

(Received 4 January 2021; revised 26 April 2021; accepted 22 July 2021)

The modelling of natural convection in porous media is receiving increased interest due to its significance in environmental and engineering problems. State-of-the-art simulations are based on the classic macroscopic Darcy-Oberbeck-Boussinesq (DOB) equations, which are widely accepted to capture the underlying physics of convection in porous media provided the Darcy number, $D a$, is small. In this paper we analyse and extend the recent pore-resolved direct numerical simulations (DNS) of Gasow et al. (J. Fluid Mech, vol. 891, 2020, p. A25) and show that the macroscopic diffusion, which is neglected in $\mathrm{DOB}$, is of the same order (with respect to $D a$ ) as the buoyancy force and the Darcy drag. Consequently, the macroscopic diffusion must be modelled even if the value of $D a$ is small. We propose a 'two-length-scale diffusion' model, in which the effect of the pore scale on the momentum transport is approximated with a macroscopic diffusion term. This term is determined by both the macroscopic length scale and the pore scale. It includes a transport coefficient that solely depends on the pore-scale geometry. Simulations of our model render a more accurate Sherwood number, root mean square (r.m.s.) of the mass concentration and r.m.s. of the velocity than simulations that employ the DOB equations. In particular, we find that the Sherwood number $S h$ increases with decreasing porosity and with increasing Schmidt number $(S c)$. In addition, for high values of $R a$ and high porosities, $S h$ scales nonlinearly. These trends agree with the DNS, but are not captured in the DOB simulations.

Key words: convection in porous media, coupled diffusion and flow, buoyancy-driven instability

$†$ Email address for correspondence: yan.jin@zarm.uni-bremen.de

(C) The Author(s), 2021. Published by Cambridge University Press. This is an Open Access article, distributed under the terms of the Creative Commons Attribution licence (http://creativecommons.org/ licenses/by/4.0/), which permits unrestricted re-use, distribution, and reproduction in any medium, provided the original work is properly cited. 


\section{S. Gasow, A.V. Kuznetsov, M. Avila and Y. Jin}

\section{Introduction}

The realization of long-term storage of $\mathrm{CO}_{2}$ in deep saline aquifers (Metz, Davidson \& De Coninck 2005; Basbug \& Gumrah 2009; Michael et al. 2009; Orr 2009; Pamukcu \& Gumrah 2009; Huppert \& Neufeld 2014), the provision of large-scale thermal-energy storage systems (Singh, Saini \& Saini 2010; Heyde \& Schmitz 2017) and the increase in the efficiency of geothermal energy extraction (Ghoreishi-Madiseh et al. 2013; Böttcher et al. 2016) are examples of emerging engineering technologies that have the potential to slow down climate change. Natural convection in porous media is a fundamental process relevant to these applications (Hewitt, Neufeld \& Lister 2012; Liang et al. 2018; Wen et al. 2018a; Hewitt 2020; Liu et al. 2020a). In general, it describes the flow of fluid in a saturated porous medium between two infinite horizontal plates driven by a temperature or species concentration difference. The variation of temperature or species concentration results in the variation of the density, which induces the buoyancy force.

In this paper, we focus on the natural convection in porous media driven by a species concentration gradient. Compared with convective heat transfer, convective mass transfer is usually characterized by high Schmidt numbers $(S c)$ and, unlike thermal energy, the mass cannot penetrate the surfaces of solid obstacles. In the absence of a porous medium, the natural convective fluid flow is governed by the dimensionless Rayleigh number, which describes the buoyancy-to-diffusion ratio (Kunes 2012). In the presence of a porous medium, a Rayleigh-Darcy number (hereafter Rayleigh number, $R a$ ) is introduced; it is a modification of the conventional Rayleigh number, which takes the effect of the porous matrix into account (Nield 1994). Mass transfer in natural convection is characterized by the Sherwood number $(S h)$, which is the ratio of the total mass transfer rate (by convection and mass diffusion) to the diffusive mass transfer rate. The onset of natural convection occurs when $S h$ exceeds unity; $S h$ quantifies the efficiency of the mass transfer enhancement due to natural convection.

Besides field research studies (Arts et al. 2008) and laboratory experiments (Kneafsey \& Pruess 2010; Faisal et al. 2015), numerical simulation is another established tool for understanding convection in porous media. Two approaches are available for the simulation of convection in porous media: pore-scale-resolving direct numerical simulations (DNS) and macroscopic (volume-averaged) simulations. Macroscopic simulations are widely employed in modelling convection in porous media (Nield \& Bejan 2017), due to their significantly lower computational costs. The first macroscopic model for fluid flow in porous media was proposed by Darcy (1856). Whitaker (1969) proposed the most commonly used macroscopic equations for the conservation of volume-averaged quantities. Using Whitaker's approach, the Darcy-Oberbeck-Boussinesq (DOB) equations can be derived, as shown in Nield \& Bejan (2017). This set of equations has often been used in recent studies; see Hewitt et al. (2012), Hewitt, Neufeld \& Lister (2013, 2014), Wen, Corson \& Chini (2015), De Paoli Zonta \& Soldati (2016) and Pirozzoli et al. (2021) as examples.

A deficiency of the DOB equations is the underlying assumption that convection in porous media is uniquely determined by the Rayleigh number, in which the pore scale is combined with the macroscopic length scale. This simplification could, however, be at the root of reported discrepancies between numerical simulations and experiments. For example, most numerical studies based on the DOB equations indicate a linear scaling of $S h$ versus $R a$ in the ultimate regime $(R a \geq 5000)$, whereas the experiments by Neufeld et al. (2010) and Keene \& Goldstein (2015) exhibited a nonlinear scaling. The experiments by Backhaus, Turitsyn \& Ecke (2011) in a Hele-Shaw cell, where the flow obeys the Darcy law but there is no porous matrix, also exhibited a nonlinear scaling. 


\section{Macroscopic two-length-scale model for natural convection}

However, recent studies showed that nonlinear scaling observed in Hele-Shaw experiments may be related to the three-dimensionality of the flow (Letelier, Mujica \& Ortega 2019; De Paoli, Alipour \& Soldati 2020). In a recent study of three-dimensional DOB simulation, Pirozzoli et al. (2021) indicated that the nonlinear scaling can occur in three-dimensional flows at very high Rayleigh numbers. This could be related to supercells at the boundary, which are the footprint of mega-plumes dominating the interior part of the flow.

Another possible reason for the nonlinear scaling is related to non-Darcy effects induced by the porous matrix. Various studies have been performed to analyse non-Darcy effects in natural convection in porous media. For example, Shao et al. (2016) and Wang \& Tan (2009) included the Brinkman term (which is a Laplacian term that is included to model the effect of macroscopic velocity gradients on the momentum transport) in their simulations of convection at low $R a$ numbers $(R a \leq 5000)$. However, the study of Vasseur, Wang \& Sen (1989) concluded that the Brinkman term is significant only for large Darcy numbers. Mijic, Laforce \& Muggeridge (2014) and Das et al. (2016) included the Forchheimer term in their models to account for the effect of turbulence. In recent years, increasing attention has been paid to hydrodynamic dispersion in porous media; see Hidalgo \& Carrera (2009), Ghesmat, Hassanzadeh \& Abedi (2011), Yang \& Vafai (2011), MacMinn et al. (2012), Wang et al. (2016), Liang et al. (2018), Wen, Chang \& Hesse (2018b), Fahs et al. (2020), Jouybari, Lundström \& Hellström (2020) and Liu et al. $(2020 b)$. It is sometimes also referred to as thermal dispersion for heat transfer problems (Pedras \& de Lemos 2008), or mass dispersion for mass transfer problems (Mesquita \& de Lemos 2004). A Fickian dispersion tensor introduced by Bear (1961) is often used to model the hydrodynamic dispersion. These studies show that hydrodynamic dispersion can have significant effects on convection in porous media, at least for high-Darcy-number problems. Gelhar, Welty \& Rehfeldt (1992), Neuman (1990) and Liang et al. (2018) indicated that the hydrodynamic dispersion is also important at low Darcy numbers, since dispersion at the macroscale (macrodispersivity) is dependent on the scale of the system, rather than the grain size. In a recent study, however, Zech et al. (2019) showed that dispersion at the macroscale varied widely and did not show any clear effect on the scale of solute plumes.

In the DNS, the Navier-Stokes equations coupled to a convection-diffusion equation for the species concentration (or temperature for heat transfer) are solved, whereby the smallest scale of the porous matrix is resolved. Owing to the high computational costs, this approach has so far only been used for simple geometries of porous matrices (Minkowycz et al. 2006; Torabi et al. 2017). Although DNS is too expensive for engineering applications, it is a powerful tool to gain a better understanding of the physics of convection in porous media and serves as a foundation for developing macroscopic models. Recently, we performed pore-scale-resolving DNS of natural convection in porous media composed of a simple porous matrix (Gasow et al. 2020). Our DNS results showed that the boundary layer thickness for convection in porous media is determined by the pore size instead of the Rayleigh number. This is distinctly different from classical DOB simulations (Huppert \& Neufeld 2014). We also showed that the scaling for the Sherwood number depends on the porosity and the pore-scale parameters and observed that the scaling law becomes nonlinear for porous media with sufficiently high porosity. Furthermore, the computed flow patterns exhibited motions with large length scales, close to the size of the whole domain, which were not found in DOB simulations. In another recent numerical study, Liu et al. (2020a) observed that the Nusselt number increases with a decrease in the porosity, while the Rayleigh-Darcy number is kept constant. 


\section{S. Gasow, A.V. Kuznetsov, M. Avila and Y. Jin}

This trend cannot be captured by the DOB equations. Liu et al. (2020a) also indicated that the ratio of the pore scale to the thickness of the thermal boundary layer has a significant effect on the scaling of the Nusselt number versus $R a$. A scaling crossover occurs when the thickness of the thermal boundary is comparable to the pore scale. Therefore, the discrepancy between the DOB solutions and the experiments could arise due to pore-scale effects.

In this paper, we develop a new macroscopic model for natural convection in porous media, which accounts for pore-scale effects. Our model is based on a detailed analysis of the DNS simulations of Gasow et al. (2020) and additional DNS carried out here. The model involves a coefficient that depends solely on the pore-scale geometry. This coefficient must be determined a priori. For each pore-scale geometry, this coefficient is determined with a single DNS performed with a fixed set of parameters. Subsequently, we show that the simulations of the model agree with our DNS results (e.g. results with respect to the Sherwood number, mean species concentration, root-mean-square (r.m.s.) species concentration and velocity) in wide ranges of pore size, Rayleigh, Schmidt and Darcy numbers.

\section{Governing equations and numerical methods}

We consider natural convection in a porous medium domain bounded by two walls (figure 1), which is the porous equivalent to the classical Raleigh-Bénard cell (Hewitt 2020). The computational domain is two-dimensional, and it has a width-to-height ratio $L / H=2$. Two different geometries of the generic porous matrix are studied. They are composed of aligned (figure $1 b$ ) or staggered (figure $1 c$ ) square obstacles. The analysis in this study is mainly based on the results of the first porous matrix, while the sensitivity of our model coefficient to the pore-scale geometry is examined with the second porous matrix. In both cases, the periodically arranged square obstacles with the size $d$ are a distance $s$ apart in the horizontal and vertical directions. The geometry of a representative elementary volume (REV) of the simulated porous medium is a square with a side length $s$, containing one obstacle.

Constant species concentrations, $c_{1}$ and $c_{0}$, are maintained at the upper and lower walls of the domain, respectively. The difference of the species concentrations at the upper and lower walls leads to density differences, which drive natural convection in the domain. The horizontal boundary conditions are periodic, whereas the no-slip boundary condition is used at the upper and lower walls and on the surfaces of the obstacles. And because mass cannot penetrate the solid matrix of the porous medium, no mass transfer is assumed at the interface, hence homogeneous Neumann boundary conditions are used at the obstacles for the species concentration. Similar set-ups have been adopted in other numerical studies of convection in porous media; see Javaheri, Abedi \& Hassanzadeh (2010), Hewitt et al. (2012), Wen et al. (2018b), and Hewitt (2020) as examples.

\subsection{Governing equations for DNS}

DNS studies were performed to gain insights into the physics of natural convection in the porous medium, to determine the coefficients for the macroscopic model, as well as to obtain the validation data. The governing equations for DNS of natural convection in porous media are the Navier-Stokes equations and the species transport equation. In the flow field, the local species concentration differences are small; hence, the Boussinesq approximation is used to account for the buoyancy force (Herwig 2013). Using Einstein's 

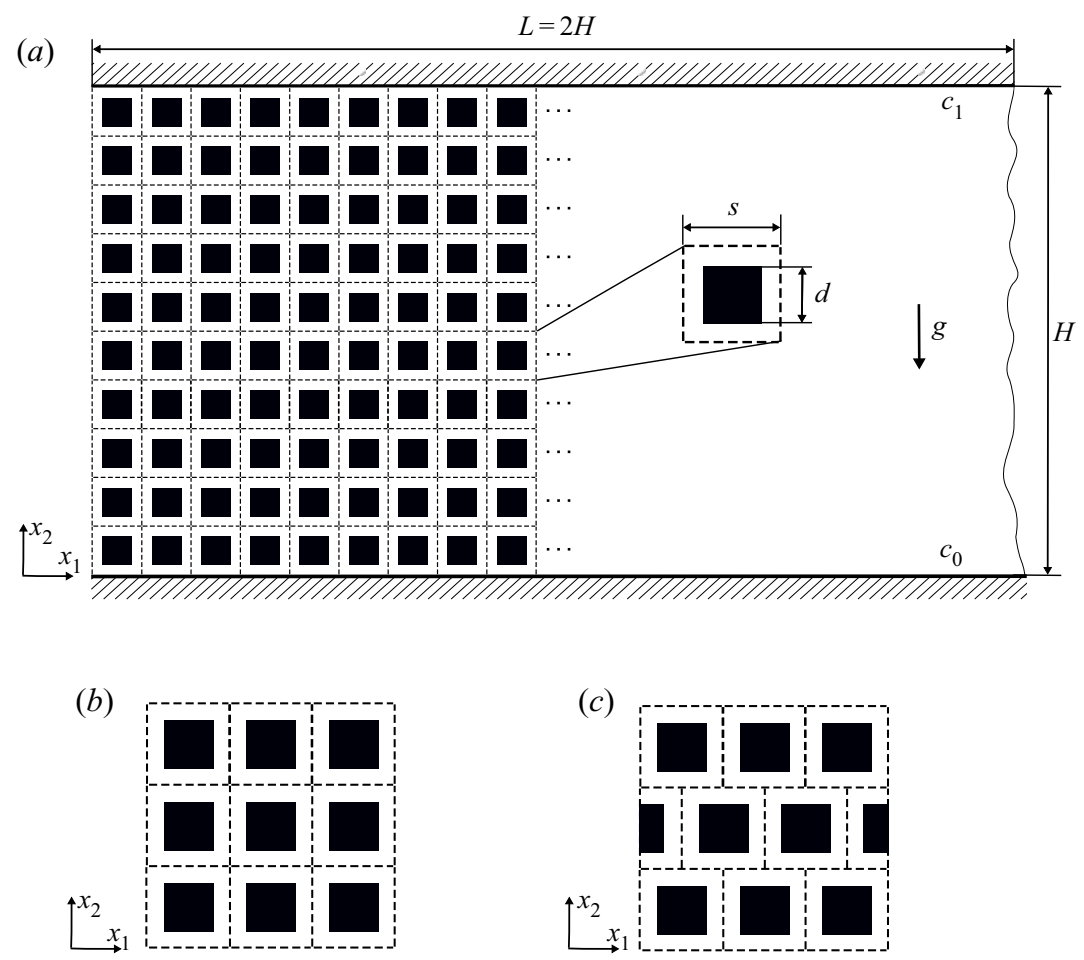

Figure 1. Structure of the computational domain occupied by a regular porous matrix, with a magnified view of a single REV, used for the DNS $(a)$. A constant species concentration difference at the top and bottom walls and periodic boundary conditions in the horizontal direction are utilized. The porous matrix inside the domain is composed of aligned $(b)$ or staggered square obstacles $(c)$.

summation convention, the governing microscopic equations for natural convection in porous media are as follows:

$$
\begin{gathered}
\frac{\partial u_{i}}{\partial x_{i}}=0 \\
\frac{\partial u_{i}}{\partial t}+\frac{\partial\left(u_{i} u_{j}\right)}{\partial x_{j}}=-\frac{\partial p}{\partial x_{i}}+v \frac{\partial^{2} u_{i}}{\partial x_{j}^{2}}+\beta g_{i}\left(c-c_{0}\right), \\
\frac{\partial c}{\partial t}+\frac{\partial\left(u_{i} c\right)}{\partial x_{i}}=D_{f} \frac{\partial^{2} c}{\partial x_{j}^{2}}
\end{gathered}
$$

where $v, D_{f}, u_{i}, p, g_{i}$ and $c$ are the kinematic viscosity, the mass diffusivity, the $i$ th component of the velocity vector, the pressure, the $i$ th component of the gravity vector and the species concentration, respectively. The concentration expansion coefficient is defined as $\beta=\beta\left(c_{0}\right)=-\left(1 / \rho_{0}\right)(\partial \rho / \partial c)_{c_{0}}$ (see Herwig \& Moschallski, 2009), where $\rho$ is the fluid density.

The Sherwood number $S h$ is calculated from the DNS as the ratio of the total mass transfer rate $\dot{m}$ (by convection and diffusion) to the mass transfer rate $\dot{m}_{\text {diff }}$ (by diffusion 


\section{S. Gasow, A.V. Kuznetsov, M. Avila and Y. Jin}

only) across the lower or upper wall (Baehr \& Stephan 2006):

$$
S h=\frac{\dot{m}}{\dot{m}_{\text {diff }}}=\frac{\int_{w} \overline{\frac{\partial c}{\partial x_{2}}} \mathrm{~d} A}{\left.\int_{w} \overline{\frac{\partial c}{\partial x_{2}}}\right|_{R a_{f}=0} \mathrm{~d} A},
$$

where the bar denotes the time-averaging operator, while the subscript $w$ denotes either the upper or lower wall surface.

\subsection{Macroscopic equations}

The macroscopic equations are obtained by averaging the Navier-Stokes equations and the species transport equations (2.1)-(2.3) over each REV (see figure 1). This method of averaging is similar to that used in de Lemos (2012); however, de Lemos (2012) carried out a time and volume averaging over each respective REV, while we performed only volume averaging. The macroscopic equations read:

$$
\begin{aligned}
& \frac{\partial \breve{u}_{i}}{\partial \breve{x}_{i}}=0, \\
& \frac{\partial \breve{u}_{i}}{\partial \breve{t}}+\frac{\partial\left(\breve{u}_{i} \breve{u}_{i} / \phi\right)}{\partial \breve{x}_{j}}+\frac{\partial\left(\phi\left\langle{ }^{i} u_{i}{ }^{i} u_{j}\right\rangle^{i}\right)}{\partial \breve{x}_{j}}=-\frac{\partial\left(\phi\langle p\rangle^{i}\right)}{\partial \breve{x}_{i}}+v \frac{\partial^{2} \breve{u}_{i}}{\partial \breve{x}_{j}^{2}}-\phi \beta g_{i}\left(\breve{c}-c_{0}\right)-\phi \breve{R}_{i} \text {, } \\
& \frac{\partial(\phi \breve{c})}{\partial \breve{t}}+\frac{\partial\left(\breve{u}_{i} \breve{c}\right)}{\partial \breve{x}_{i}}+\frac{\partial\left(\phi\left\langle{ }^{i} u_{i}{ }^{i} c\right\rangle\right.}{\left.\partial{ }^{i}\right)}=D_{m} \frac{\partial^{2} \breve{c}}{\partial \breve{x}_{j}},
\end{aligned}
$$

where the sign $\smile$ denotes an REV-averaged quantity. The operator \langle\rangle$^{i}$ denotes the intrinsic volume averaging in the fluid phase, which is adopted from Whitaker (1986). The left superscript $i$ denotes the intrinsic deviation of a volume-averaged quantity, e.g. ${ }^{i} u_{i}=u_{i}-\left\langle u_{i}\right\rangle^{i}$. The porosity $\phi$ is defined as $\phi=V_{\text {void space }} / V_{\text {total }}, u_{i}=\phi\left\langle u_{i}\right\rangle^{i}$ is the volume-averaged velocity, which is often referred to as the superficial velocity, and $\breve{c}=\langle c\rangle^{i}$ is the intrinsic averaged mass concentration. The subscript $m$ denotes an effective property in the volume-averaged equations, e.g. $D_{m}$ is the effective mass diffusivity. Simulations of small domains are needed to determine the value of $D_{m}$ for a specific pore-scale geometry (see Gasow et al. 2020).

The terms $\phi\left\langle{ }^{i} u_{i}{ }^{i} u_{j}\right\rangle^{i}, \phi\left\langle{ }^{i} u_{i}{ }^{i} c\right\rangle^{i}$ and $\breve{R}_{i}$ are the momentum dispersion, mass dispersion and total drag, respectively. The momentum and mass dispersion terms have been neglected in our model due to the underlying assumptions for convection in porous media with low Darcy numbers (see Appendix A1). Since the local Reynolds number $\operatorname{Re}_{K}=|\breve{\boldsymbol{u}}| \sqrt{K} / \nu$ in our simulations is generally smaller than unity (Gasow et al. 2020), the Forchheimer term in $R_{i}$ can also be neglected (Nield \& Bejan 2017). The effects of the macroscopic velocity gradient on $\widetilde{R}_{i}$ can be modelled with a Laplacian term, which was first proposed by Brinkman (1949) and then was extensively studied and improved; see Rao, Kuznetsov \& Jin (2020), Zaripov, Mardanov \& Sharafutdinov (2019), Zhao et al. (2018), Liu, Patil \& Narusawa (2007), Valdes-Parada, Ochoa-Tapia \& Alvarez-Ramirez (2007), Vafai (2005), Starov \& Zhdanov (2001) and Ochoa-Tapia \& Whitaker (1995) as examples. Here, we 


\section{Macroscopic two-length-scale model for natural convection}

model the sum of the total drag $\breve{R}_{i}$ and the diffusion term $v\left(\partial^{2} \breve{u}_{i} / \partial \breve{x}_{j}^{2}\right)$ in (2.6) as

$$
v \frac{\partial^{2} \breve{u}_{i}}{\partial \breve{x}_{j}^{2}}+\breve{R}_{i}=\frac{v}{K} \breve{u}_{i}+v_{m} \frac{\partial^{2} \breve{u}_{i}}{\partial \breve{x}_{j}^{2}},
$$

where $K$ and $v_{m}$ are the permeability and effective viscosity of the porous medium. Simulations of small domains are needed to determine their values a priori (see Gasow et al. (2020) for details of how they were determined). The macroscopic momentum equation (2.6) is hence simplified to

$$
\frac{\partial \breve{u}_{i}}{\partial \breve{t}}+\frac{\partial\left(\breve{u}_{i} \breve{u}_{i} / \phi\right)}{\partial \breve{x}_{j}}=-\frac{\partial\left(\phi\langle p\rangle^{i}\right)}{\partial \breve{x}_{i}}+v_{m} \frac{\partial^{2} \breve{u}_{i}}{\partial \breve{x}_{j}^{2}}-\phi \beta g_{i}\left(\breve{c}-c_{0}\right)-\phi \frac{v}{K} \breve{u}_{i} .
$$

\subsection{Two-length-scale diffusion assumption}

Normalizing the governing equations (2.5), (2.9) and (2.7) using the characteristic concentration difference $\Delta c=c_{1}-c_{0}$, velocity $u_{m}=\beta \Delta c g K / \nu$, length $H$ and time $t_{m}=H / u_{m}$, the following dimensionless macroscopic equations are obtained:

$$
\begin{gathered}
\frac{\partial \hat{u}_{i}}{\partial \hat{x}_{i}}=0, \\
\frac{\partial \hat{u}_{i}}{\partial \hat{t}}+\frac{\partial\left(\hat{u}_{i} \hat{u}_{i} / \phi\right)}{\partial \hat{x}_{j}}=-\frac{\partial\left(\phi\langle\tilde{p}\rangle^{i}\right)}{\partial \hat{x}_{i}}+\frac{a_{v} S c}{\gamma_{m} R a} \frac{\partial^{2} \hat{u}_{i}}{\partial \hat{x}_{j}^{2}}-\frac{\phi S c}{\gamma_{m} R a D a} z_{i} \hat{c}-\frac{\phi S c}{\gamma_{m} R a D a} \hat{u}_{i}, \\
\frac{\partial(\phi \hat{c})}{\partial \hat{t}}+\frac{\partial\left(\hat{u}_{i} \hat{c}\right)}{\partial \hat{x}_{i}}=\frac{1}{R a} \frac{\partial^{2} \hat{c}}{\partial \hat{x}_{j}^{2}} .
\end{gathered}
$$

Here ${ }^{\wedge}$ denotes a dimensionless volume-averaged quantity, $\hat{c}$ is the dimensionless volume-averaged species concentration defined as $\hat{c}=\left(\langle c\rangle^{i}-c_{0}\right) /\left(c_{1}-c_{0}\right), a_{v}=v_{m} / v$ is the ratio of the effective viscosity $v_{m}$ to the molecular viscosity of the fluid $v$, and $\gamma_{m}=D_{m} / D_{f}$ is the ratio of the effective mass diffusivity $D_{m}$ to the mass diffusivity of the fluid $D_{f}$.

The Rayleigh number in (2.11) and (2.12) is defined by using the common definition of this parameter for natural convection in porous media, as in Nield (1994):

$$
R a \equiv \frac{R a_{f} D a}{\gamma_{m}}=\frac{H \beta \Delta c g K}{D_{m} v} .
$$

The Schmidt number is defined as

$$
S c=\frac{v}{D_{f}}
$$

The Darcy number is defined as

$$
D a=\frac{K}{H^{2}} .
$$

By assuming that $a_{v}$ is independent of $\mathrm{Da}$ and taking the leading-order terms with respect to $1 / D a$ in $(2.11)$, one obtains the well-known DOB equations. However, we reported in our recent DNS study (Gasow et al. 2020) that the boundary layer thickness is determined by the pore size, which is characterized by $\sqrt{K}$. In addition, similar profiles for temporally 


\section{S. Gasow, A.V. Kuznetsov, M. Avila and Y. Jin}

and horizontally averaged quantities are observed when the distance from the wall is normalized with the pore size. Therefore, the Laplacian term $\left(a_{\nu} S c / \gamma_{m} R a\right)\left(\partial^{2} \hat{u}_{i} / \partial \hat{x}_{j}^{2}\right)$ in (2.11) is expected to scale as $1 / K$ and should be of order $1 / D a$, exactly as the Darcy term $-\left(\phi S c / \gamma_{m} R a D a\right) \hat{u}_{i}$ and the buoyancy force term $-\left(\phi S c / \gamma_{m} R a D a\right) z_{i} \hat{c}$. Hence, the Laplacian term in the macroscopic equation cannot be neglected even if the Darcy number is small.

We here propose a model for the effective viscosity $v_{m}$ based on a two-length-scale diffusion (TLSD) hypothesis, in which the macroscopic diffusion is determined by the pore size, characterized by $\sqrt{K}$, and the distance between the lower and upper boundaries $H$. Our TLSD hypothesis is supported by our recent DNS (Gasow et al. 2020), where we showed that natural convection in porous media is determined by these two length scales. The pore size characterizes the boundary layer thickness and the size of proto-plumes, whereas the distance between the two walls determines the size of mega-plumes.

Based on the TLSD assumption stated above, $a_{v}$ is modelled as

$$
a_{v}=\frac{v_{m}}{v}=\frac{a_{v}^{*}}{D a},
$$

where $a_{v}^{*}$ is a constant assumed to be solely determined by the pore-scale geometry of the porous matrix. Note that the two length scales $\sqrt{K}$ and $H$ are combined in $D a$. At the upper and lower walls, we imposed constant species concentrations, $c_{1}$ and $c_{0}$, respectively, and the no-slip boundary condition.

It should be noted that only the leading-order terms of $D a$ for diffusion are kept in the macroscopic equations (2.10)-(2.12). As $D a \rightarrow 0$, the macroscopic governing equations can be further simplified to

$$
\begin{gathered}
\frac{\partial \hat{u}_{i}}{\partial \hat{x}_{i}}=0, \\
\frac{\partial \hat{p}}{\partial \hat{x}_{i}}+\hat{c} z_{i}+\hat{u}_{i}=\frac{a_{v}^{*}}{\phi} \frac{\partial^{2} \hat{u}_{i}}{\partial \hat{x}_{j}^{2}}, \\
\frac{\partial \hat{c}}{\partial \hat{t}^{*}}+\frac{\partial\left(\hat{u}_{i} \hat{c}\right)}{\partial \hat{x}_{i}}=\frac{1}{R a} \frac{\partial^{2} \hat{c}}{\partial \hat{x}_{j}^{2}},
\end{gathered}
$$

where $\hat{p}=\operatorname{RaD} a\langle\tilde{p}\rangle^{i} / \gamma_{m} S c$ is the normalized pressure. The dimensionless time is modified to be $\hat{t}^{*}=\breve{t} / \phi$. These macroscopic equations (2.17)-(2.19) become the DOB equations if $a_{v}^{*}$ is set to zero. When the DOB equations are solved, only the velocity component in the wall-normal direction is set to zero at the upper and lower walls. This boundary condition was also used in other DOB simulations; see Hewitt et al. (2014) and Wen et al. (2015) as examples. In this paper, the macroscopic simulations were carried out by solving equations (2.10)-(2.12), so that the effect of the Darcy number can be assessed. The Sherwood number for the macroscopic model simulations is defined using the same definition as for the DNS, which is given in (2.4).

\subsection{Numerical method}

For the simulations, a finite-volume method (FVM) was utilized. The solvers were developed by using the open-source code package OpenFoam 6. The spatial discretization was implemented by a second-order central-difference scheme. For time derivatives, 


$\begin{array}{ll}s / d & 1.175-5 \\ \phi & 0.28-0.96 \\ \gamma_{m} & 0.16-0.92 \\ a_{v}^{*} & 2.4 \times 10^{-9}-5.18 \times 10^{-5} \\ H / s & 10-100 \\ D a & 7 \times 10^{-8}-1.7 \times 10^{-4}\end{array}$

Table 1. Ranges of geometrical parameters for the studied test cases.

the second-order implicit backward method was used. For the correction and coupling of the pressure and velocity fields, the pressure-implicit scheme with splitting of operators (PISO) algorithm was used (Versteeg \& Malalasekera 2007). A stabilized preconditioned (bi)conjugate gradient solver was utilized to solve the pressure field and the momentum and species concentration equations. We have performed the code validation for our DNS solver extensively in our previous studies (Jin et al. 2015; Uth et al. 2016; Jin \& Kuznetsov 2017; Gasow et al. 2020).

\section{Studied test cases}

\subsection{Description of the test cases}

We continued selected DNS cases of Gasow et al. (2020) to improve the statistics and thus to allow a more thorough validation of our hypothesis. In addition, we also computed these cases by solving the macroscopic equations (2.10)-(2.12) with our two-length-scale diffusion model. The Rayleigh numbers $R a$ are up to 20000 and the Schmidt numbers Sc are 1 and 250. The ranges of geometrical parameters of the studied test cases are given in table 1 . For both DNS and macroscopic simulation cases $u_{i}=0$ and $c=\left(c_{1}-c_{0}\right) / 2$ were used as initial conditions.

To obtain representative statistical results, the time averaging, denoted by the bar over, of the respective variable was performed after the flow and mass concentration fields reached a statistically steady state. As an example, the time evolutions of the instantaneous Sherwood number for the DNS case with $H / s=100, s / d=1.5, R a=20000$ and $S c=250$ are shown in figure 2. The time averaging of the Sherwood number has been started after the time marked by the red dashed line. At least 200 dimensionless time units $H / u_{m}$ are calculated to obtain the statistical results.

\subsection{Determination of the model coefficient}

The coefficient $a_{v}^{*}$ for a specific pore-scale geometry cannot be computed a priori with simulations of small domains (as for the other model parameters). Here, we empirically determine $a_{v}^{*}$ by simulating natural convection within the specific pore-scale geometry with fixed values of $H / s, S c$ and $R a$. Since we only keep the leading-order terms of the order $D a$ in our model equations, a test case with a sufficiently small Darcy number should be used to ensure that the higher-order terms of $D a$ are negligible. In particular, we performed a parametric study for $a_{v}^{*}(\phi)$ while keeping $H / s=20, S c=250$ and $R a=20000$ fixed. These parameter values were selected because the Sherwood number from DNS marginally changes as $H / s$ is increased (i.e. as the pore size is decreased); hence the effect of higher-order terms of $D a$ on $S h$ can be safely neglected. The value of $a_{v}^{*}$ is selected for each considered porosity value, so that the Sherwood number from the 


\section{S. Gasow, A.V. Kuznetsov, M. Avila and Y. Jin}

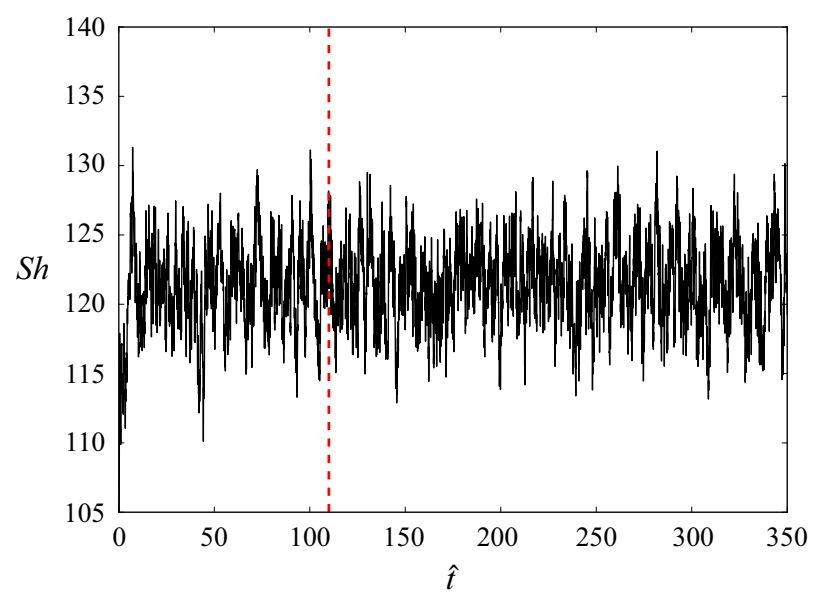

Figure 2. The time evolution of the instantaneous Sherwood number for the DNS case with $H / s=50, s / d=$ $1.5, R a=20000$ and $S c=250$. The dashed red line marks the time at which the time averaging is started; and $\hat{t}=t u_{m} / H$ is the dimensionless time.

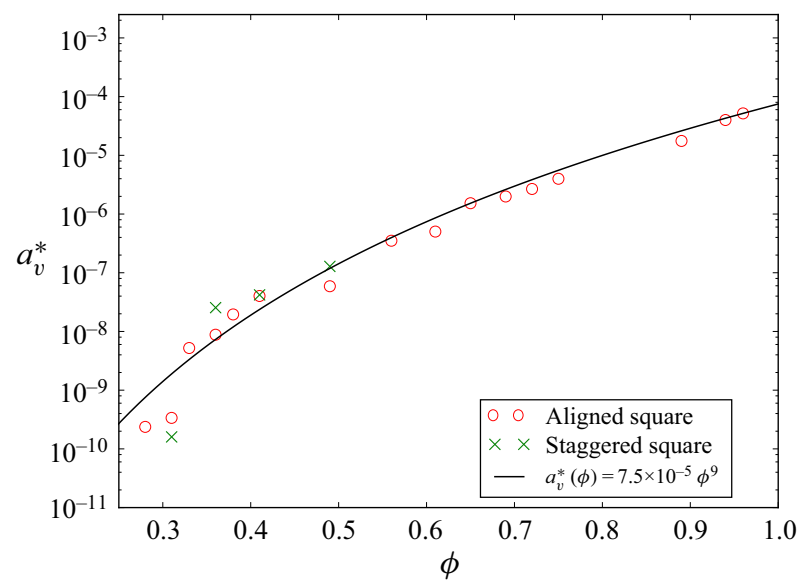

Figure 3. Dependence of the model coefficient $a_{v}^{*}$ on the porosity $\phi$, for porous matrices composed of aligned and staggered square obstacles.

macroscopic simulation matches the DNS results. Figure 3 shows the dependence of $a_{v}^{*}$ on the porosity $\phi$.

We expect that $a_{v}^{*}$ is a geometrical parameter that is independent of $S c, R a$ and $D a$. This will be examined later in $\S 5$. The value of $a_{v}^{*}$ only mildly changes when the porous matrix is switched from aligned squares to staggered squares. According to our DNS results, $a_{v}^{*}$ can be well correlated by

$$
a_{v}^{*}(\phi)=7.5 \times 10^{-5} \phi^{9}
$$

This correlation has reasonable accuracy for $0.28<\phi<0.95$. However, the variations of pore-scale geometries used in this study are limited. In particular, the flow structures in randomly packed porous matrices may be distinctly different from those in regularly 


$\begin{array}{lllll}\text { ID } & \text { Mesh resolution }\left(N_{x} \times N_{y}\right) & \text { Mesh size } & \text { Comax } & S h \\ \text { a } & 800 \times 1200 & 960000 & 0.2 & 105.3 \\ \text { b } & 1000 \times 1600 & 1600000 & 0.2 & 110.1 \\ \text { c } & 2000 \times 3000 & 6400000 & 0.2 & 120.1 \\ \text { d } & 3200 \times 2200 & 7040000 & 0.2 & 122 \\ \text { e } & 2000 \times 3200 & 6400000 & 0.6 & 122.4 \\ f & 2000 \times 3200 & 6400000 & 0.8 & 121.5 \\ \text { g } & 2000 \times 3200 & 6400000 & 1.1 & 115.8\end{array}$

Table 2. Influence of mesh and time step on the Sherwood number $S h$. The test case with $H / s=50, s / d=1.5$, $R a=20000$ and $S c=250$ is used in the parametric study. The cases c, d, e and $\mathrm{f}$ are considered to be meshand time-step-independent. The mesh resolution and maximum Courant number of the case $\mathrm{f}$ (in italic) are used in all cases of macroscopic simulation.

packed porous matrices (Liu et al. 2020a). Studies with more pore-scale geometries are needed to test the generality of (3.1).

\subsection{Mesh and time-step independence studies}

The mesh and time-step independence studies for the DNS cases have already been performed in our previous work (see Gasow et al. 2020). Here we focus on the influence of the mesh and time step on the macroscopic simulation results (solution of (2.10)-(2.12)). The numerical results for the Sherwood number are shown in table 2. At least 200 dimensionless time units $H / u_{m}$ were calculated to obtain the statistical results.

The results of the resolution study show that the Sherwood number is under predicted if the mesh resolution is too low (see table 2 cases a and b) or the maximum Courant number is too high (see table 2 case g). According to the mesh/time-step independence study, $C o_{\max }=0.8$ and mesh resolution $2000 \times 3200$ (case f) were used for all cases of macroscopic simulation. The numerical error of $S h$ in the macroscopic simulations is estimated to be $2.8 \%$, which is the maximum variation of $S h$ in the cases $\mathrm{c}, \mathrm{d}$, e and $f$. All simulations were performed on the clusters of the HLRN (North-German Supercomputing Alliance), using $2 \times$ Intel Cascade Lake Platinum 9242 CPUs (CLX-AP) with 96 cores per node. The DNS cases use up to $7.2 \times 10^{7}$ mesh cells, which requires a parallel computing time of 1200 hours using 384 processors. The macroscopic simulation cases use up to $6.4 \times 10^{6}$ mesh cells.

\section{DNS results}

In this section, we focus on an a priori verification of the TLSD hypothesis. The model results are compared with the DNS results in $\S 5$.

\subsection{Budget of the macroscopic kinetic energy}

The budget for the time- and line-averaged macroscopic kinetic energy $\langle\bar{K}\rangle^{x 1}=\frac{1}{2}\left\langle u_{i}^{2}\right\rangle^{x 1}$ was calculated from the DNS for $R a=20000, s / d=1.5, s / d=1.25, S c=250$ and $D a$ in the range $3.5 \times 10^{-7}$ to $3.5 \times 10^{-5}$. By averaging the momentum equation (2.2) over REVs, taking the dot product with the superficial velocity $\breve{u}_{i}=\phi\left\langle u_{i}\right\rangle^{i}$, and then averaging 


\section{S. Gasow, A.V. Kuznetsov, M. Avila and Y. Jin}

in time and in the horizontal direction $x_{1}$, we obtained the following equation for $\langle\bar{K}\rangle^{x 1}$ :

$$
\begin{aligned}
& \left.-\left\langle\overline{\breve{u}_{i} \phi\left\langle\frac{\partial\left(u_{i} u_{j}\right)}{\partial x_{j}}\right\rangle^{i}}\right\rangle^{x 1}-\overline{\left\langle\breve{u}_{i} \phi\left\langle\frac{\partial p}{\partial x_{i}}\right\rangle^{i}\right.}\right\rangle^{x 1}+\left\langle\overline{\left\langle\breve{u}_{i} \phi\left\langle v \frac{\partial^{2} u_{i}}{\partial x_{j}^{2}}\right\rangle^{i}\right.}\right\rangle^{x 1} \\
& \left.+\overline{\left\langle\breve{u}_{i} \phi\left\langle\beta g_{i}\left(c-c_{0}\right)\right\rangle^{i}\right.}\right\rangle^{x 1}=0 .
\end{aligned}
$$

Equation (4.1) shows that the budget for $\langle\bar{K}\rangle^{x 1}$ includes:

- the production by the buoyancy force, $K_{\text {buoy }}=\left\langle\overline{\left\langle\bar{u}_{i} \phi\left\langle\beta g_{i}\left(c-c_{0}\right)\right\rangle^{i}\right.}\right\rangle^{x 1}$;

- the loss due to viscous dissipation, $\left.K_{\text {diff }}=\overline{\left\langle\breve{u}_{i} \phi\left\langle v\left(\partial^{2} u_{i} / \partial x_{j}^{2}\right)\right\rangle^{i}\right.}\right\rangle^{x 1}$;

- the loss due to pressure gradient, $K_{\text {pres }}=-\left\langle\overline{\bar{u}_{i} \phi\left\langle\partial p / \partial x_{i}\right\rangle^{i}}\right\rangle^{x 1}$;

- the transport due to convection, $\left.K_{\text {conv }}=-\overline{\left\langle\bar{u}_{i} \phi\left\langle\partial\left(u_{i} u_{j}\right) / \partial x_{j}\right\rangle^{i}\right.}\right\rangle^{x 1}$.

In the DOB equations, the Darcy term (Darcy drag) is the only source of losses of macroscopic kinetic energy. The Darcy losses read

$$
K_{\text {Darcy }}=-\phi(v / K)\left\langle\overline{u_{i}^{2}}\right\rangle^{x 1}
$$

The budget of $\langle\bar{K}\rangle^{x 1}$ is studied using the test case with $s / d=1.5 \quad(\phi=0.56)$, $H / s=20, R a=20000, D a=8.8 \times 10^{-6}$ and $S c=250$. Figure 4 shows the distribution of $K_{\text {buoy }}, K_{\text {diff }}, K_{\text {pres }}$ and $K_{\text {conv }}$ in the wall-normal direction. They are normalized with the characteristic kinetic energy $K_{\text {mean }}=\frac{1}{2} u_{m}^{2}$ or $K_{\text {buoy }}$. The distance from the lower wall is normalized with the pore size $s$. It is evident that more macroscopic kinetic energy is produced by the buoyancy force in the central region than in the region close to the wall. The transport of $\langle\bar{K}\rangle^{x 1}$ due to convection is much smaller than $K_{b u o y}$, so it can be neglected. Here $-K_{\text {diff }}$ and $-K_{\text {pres }}$ are the losses of the macroscopic kinetic energy. Both $-K_{\text {diff }}$ and $-K_{\text {pres }}$ increase with increasing distance from the wall $x_{2} / s$. The loss of $\langle\bar{K}\rangle^{x 1}$ in the region close to the wall is mainly due to the pressure gradient $-K_{\text {pres }}$.

Figure 5 shows the loss of the macroscopic kinetic energy due to the Darcy drag $K_{\text {Darcy }}$ (assuming that the superficial velocity calculated from the macroscopic simulation is identical to the DNS solution). The drag $K_{\text {Darcy }}$ is normalized by $K_{\text {mean }}$ or $K_{\text {buoy }}$. It can be seen that $K_{\text {Darcy }}$ is close to $K_{\text {buoy }}$ in the region away from the wall $\left(x_{2} / s \gg 0\right)$. However, $K_{\text {Darcy }} / K_{\text {buoy }}$ is smaller than 0.85 in the first three REVs adjacent to the wall $\left(x_{2} / s<3\right)$. The DNS results confirm that the Darcy term, which accounts for the losses due to the Darcy drag, cannot account for all the losses of the macroscopic kinetic energy.

The question arises of whether the difference between $K_{\text {Darcy }}$ and $K_{\text {buoy }}$ shown in figure 5 is because the Darcy number in the DNS case is not small enough. To answer this question, $K_{\text {pres }} / K_{\text {buoy }}, K_{\text {diff }} / K_{\text {buoy }}, K_{\text {conv }} / K_{\text {buoy }}$ and $K_{\text {darcy }} / K_{\text {buoy }}$ in the first REV cell next to the bottom wall for different Darcy numbers are compared in figure 6. It is evident from this figure that all of these quantities stay almost constant as the Darcy number is decreased from $3.5 \times 10^{-5}$ to $3.5 \times 10^{-7}$, suggesting that the Darcy numbers in our DNS cases are small enough for the presented analysis. The Darcy number has a noticeable effect as it is increased to $\sim 3 \times 10^{-5}$. In this case, $K_{\text {pres }} / K_{\text {buoy }}$ and $K_{\text {Darcy }} / K_{\text {buoy }}$ become smaller, $K_{\text {diff }} / K_{\text {buoy }}$ becomes larger, whereas $K_{\text {conv }} / K_{\text {buoy }}$ is still negligibly small. We speculate that higher $K_{\text {diff }}$ at very large Darcy numbers is due to the mass dispersion, which is 

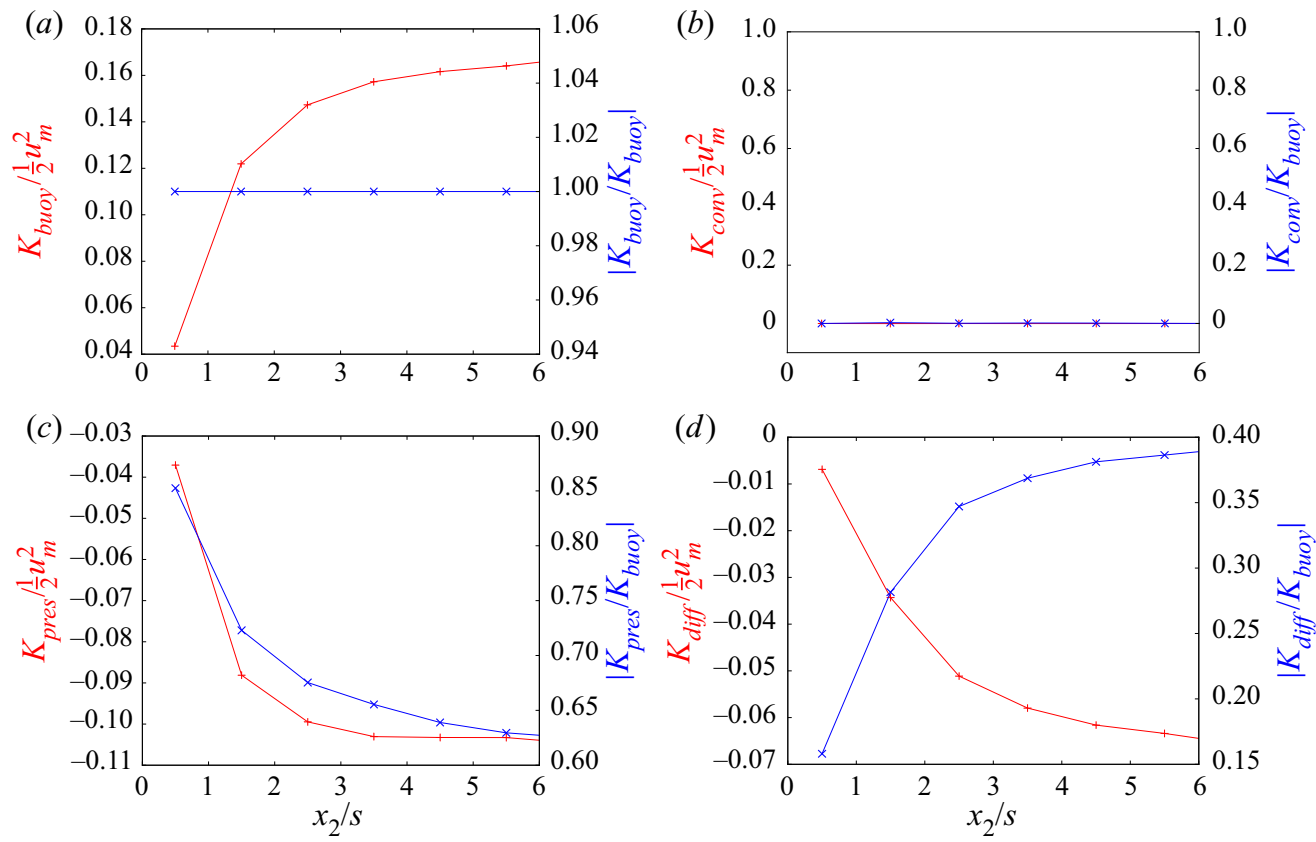

Figure 4. Distribution of the budget of the macroscopic kinetic energy $\langle\bar{K}\rangle^{x 1}$ in the wall-normal direction. Here $s / d=1.5(\phi=0.56), H / s=20\left(D a=8.8 \times 10^{-6}\right), R a=20000$ and $S c=250$.

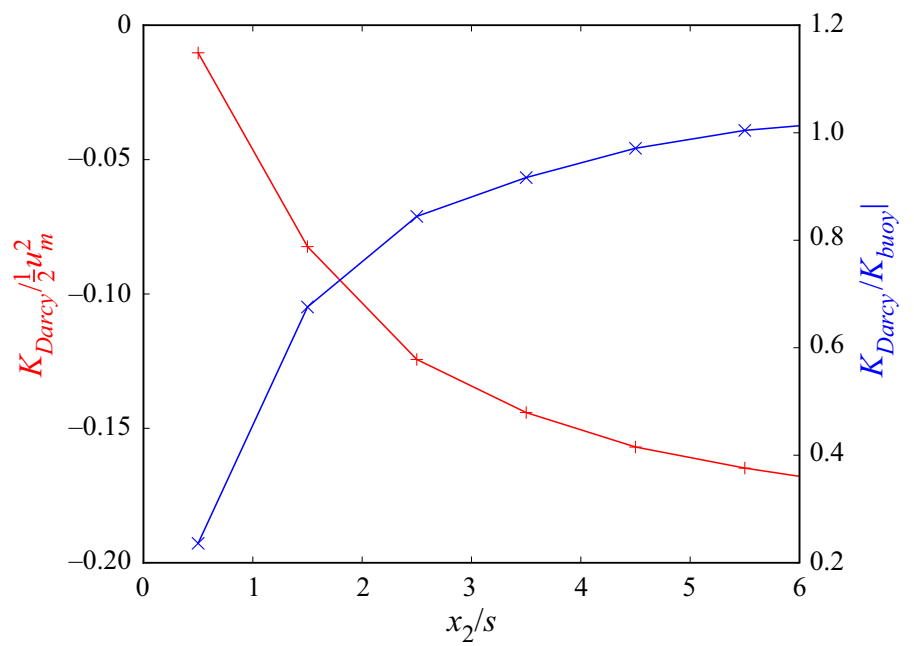

Figure 5. Distribution of the loss of the macroscopic kinetic energy $\langle\bar{K}\rangle^{x 1}$ due to the Darcy drag. Here $s / d=1.5(\phi=0.56), H / s=20\left(D a=8.8 \times 10^{-6}\right), R a=20000$ and $S c=250$.

neglected in our macroscopic model (convection with very large Darcy numbers is out of the scope of this study).

Our budget analysis shows that, in the near-wall region, there is a difference between the loss due to the Darcy drag $K_{\text {Darcy }}$ and the overall loss, which is identical to $-K_{\text {buoy }}$. 

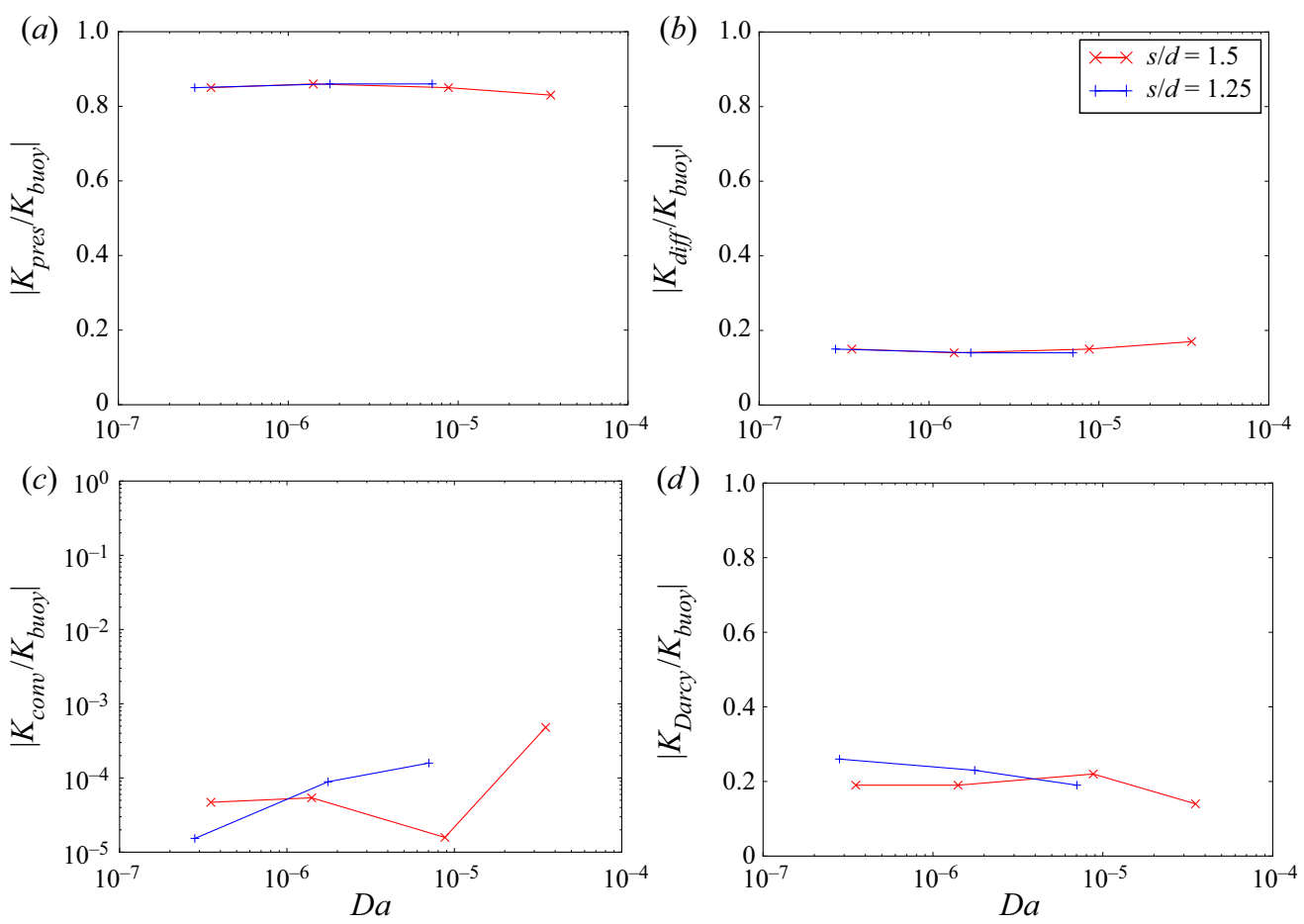

Figure 6. Plots of $K_{\text {pres }} / K_{\text {buoy }}(a), K_{\text {diff }} / K_{\text {buoy }}(b), K_{\text {conv }} / K_{\text {buoy }}(c)$ and $K_{\text {Darcy }} / K_{\text {buoy }}(d)$ in the first REV cell next to the bottom wall versus the Darcy number. Here $s / d=1.5(\phi=0.56)$ with $H / s=10,20,50,100$ and $s / d=1.25(\phi=0.36)$ with $H / s=10,20,50, R a=20000$ and $S c=250$.

Since the transport of $\langle\bar{K}\rangle^{x 1}$ is negligibly small, this suggests that another source for the loss of $\langle\bar{K}\rangle^{x 1}$ should be considered in the macroscopic equations.

\subsection{Sh-Da dependence}

According to our hypothesis, the macroscopic diffusion, the Darcy drag and the buoyancy force are of the same order with respect to the Darcy number, so the macroscopic diffusion cannot be neglected even if the Darcy number is small. To examine our hypothesis, we investigated the relationship between the Sherwood number and the Darcy number. We varied $D a$ in the range $3.5 \times 10^{-7}$ to $3.5 \times 10^{-5}$ for $s / d=1.5(\phi=0.56)$ and $2.8 \times 10^{-7}$ to $7 \times 10^{-6}$ for $s / d=1.25(\phi=0.36)$; the corresponding $H / s$ ratios are in the range 10-100. If our hypothesis were true, the Sherwood number should gradually become independent of $\mathrm{Da}$ and should not approach the DOB solution.

The DNS results shown in figure 7 generally support our assumption, i.e. the values of $S h$ for $S c=250$ depend only weakly on $D a$, when $D a$ is small enough. The values of $S h$ are also different from the DOB solution. As shown in figure 7(a), the same trend is found for $s / d=1.25(\phi=0.36)$ and $S c=1$, where $S h$ depends weakly on $D a$. The only exception is the case for $s / d=1.5(\phi=0.56)$ and $S c=1$, where $S h$ still increases with decreasing $D a$ (but it is still far away from the DOB result). Test cases with even smaller Darcy numbers could be computed to probe the Da dependence more thoroughly. However, the calculation of these cases would be extremely expensive and hence out of the scope of this study. 

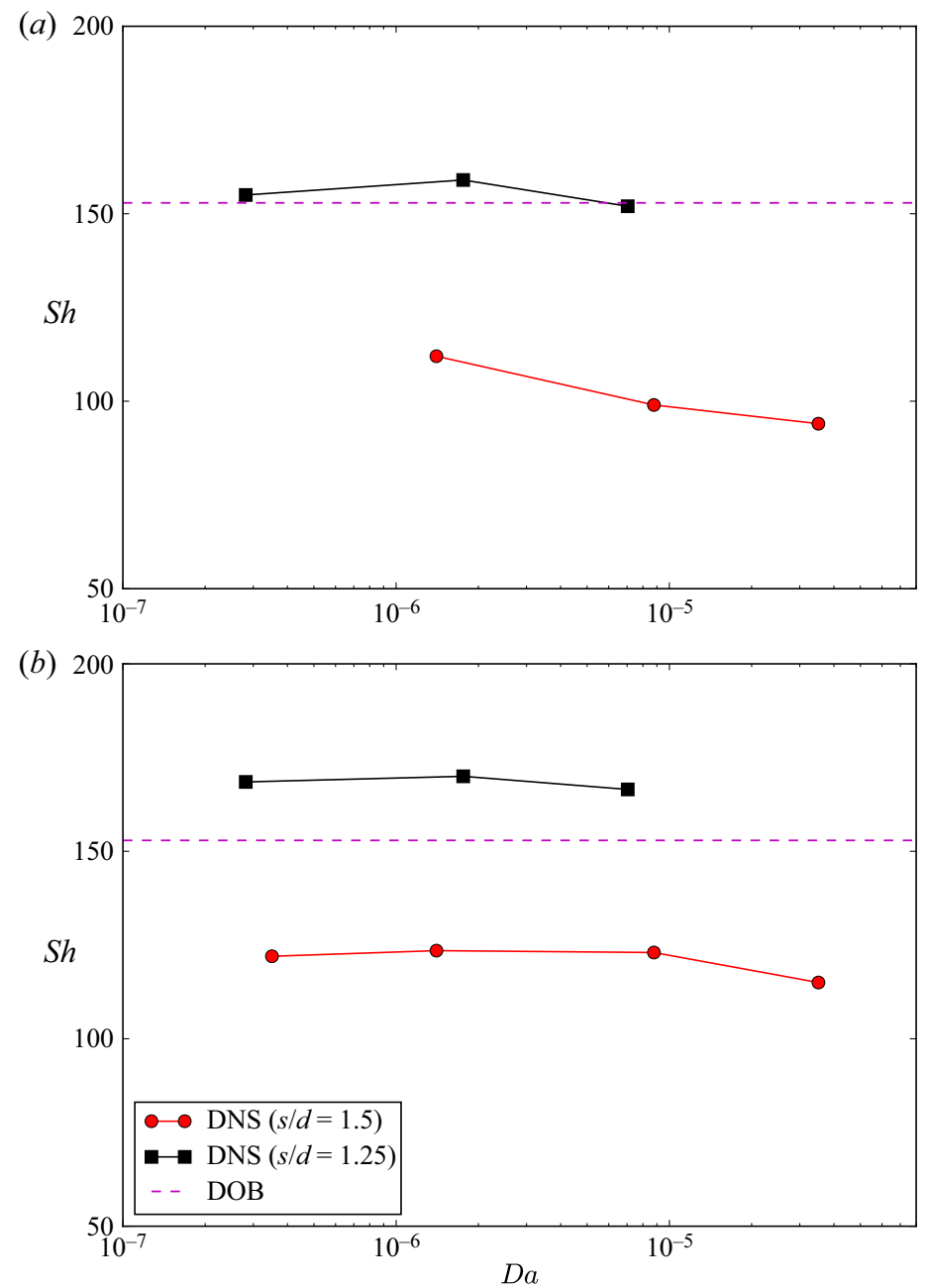

Figure 7. The $S h(D a)$ dependence for the DNS and DOB cases. Here $s / d=1.5(\phi=0.56)$ with $H / s=$ $10,20,50,100$ and $s / d=1.25(\phi=0.36)$ with $H / s=10,20,50$ and $R a=20000$, for $(a) S c=1$ and (b) $S c=250$.

It should be noted that the Darcy numbers for real applications are much smaller than the values used in the DNS cases. However, since our DNS results for the Sherwood number are approximately $D a$-independent, we expect that it is possible to predict the Sherwood numbers using DNS with relatively higher (computationally affordable) Darcy numbers.

In a recent DNS study, Liu et al. (2020a) proposed the following correlation for estimating the Nusselt number (equivalent to $S h$ in this study):

$$
S h \approx c \phi\left(\frac{H}{l}\right)^{4} S c^{2} R e_{r m s}^{2} R a_{f}^{-1}+1,
$$

where $c$ is an undetermined constant according to the work of Grossmann \& Lohse (2000, 2001, 2004), $l$ is the minimum spacing between the obstacles, $R e_{r m s}=U_{r m s} l / v$ is the Reynolds number based on the volume-averaged r.m.s. velocity magnitude, 

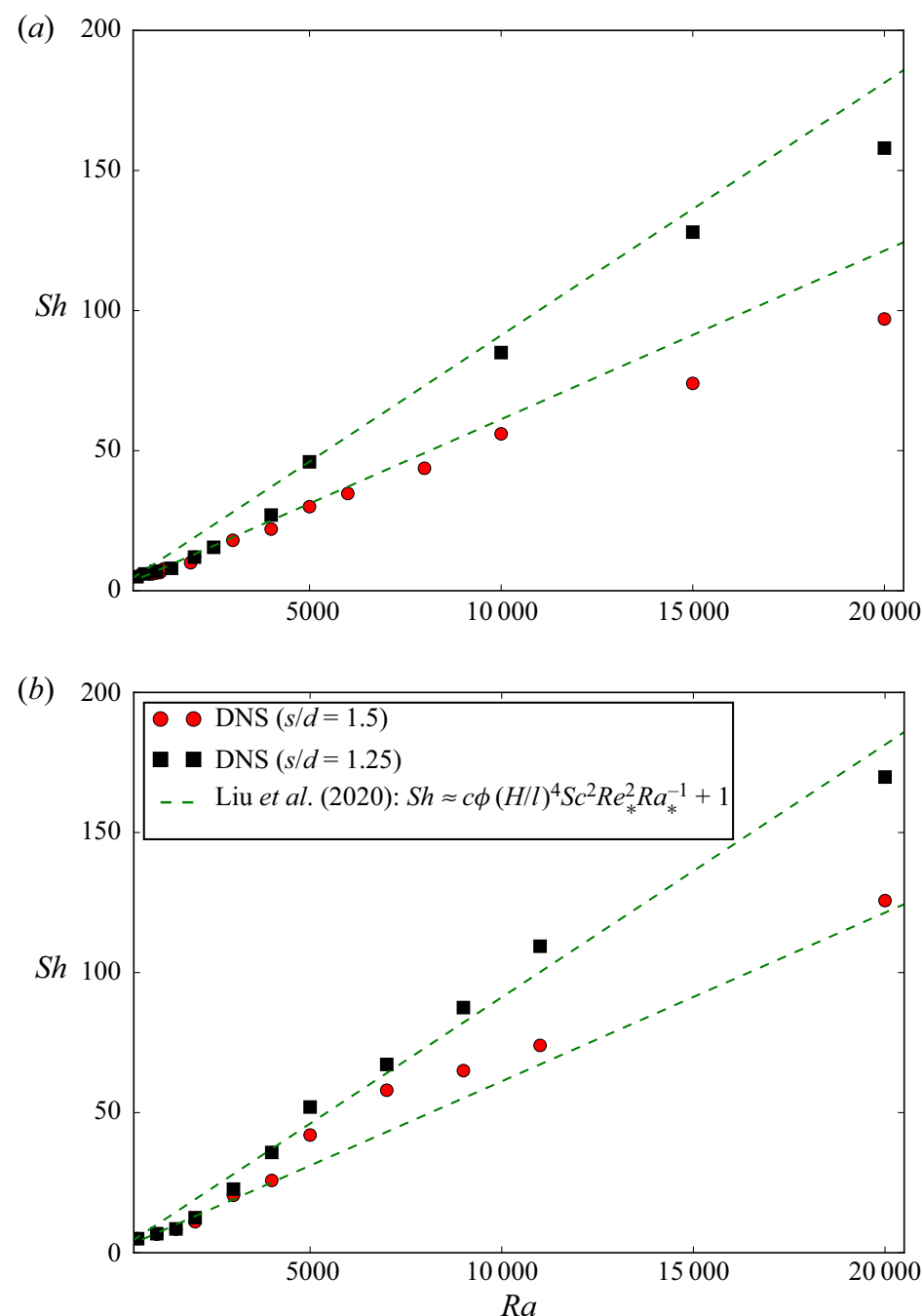

Figure 8. Sherwood number versus the Rayleigh number for $R a$ in the range 500-20000 compared to the correlation proposed by Liu et al. (2020a), with (a) $S c=1$ and (b) $S c=250$.

and $R a_{f}=H^{3} \beta \Delta c g / \nu D_{f}$ is the Rayleigh number defined for the free fluid flow. If we set the value of $c$ to 1250 and determine $U_{r m s}$ from our DNS results, the results of (4.3) are in good agreement with our DNS results for different values of $\phi$ and Sc (see figure 8). It should be noted that (4.3) is proposed based on the flow condition that viscosity dominates; hence intense kinetic energy dissipation takes place within the bulk domain and turbulence is suppressed in the pore canals. For the volume- and time-averaged kinetic energy dissipation rate $\left\langle\epsilon_{u}\right\rangle^{v, t}$, the following proportionality is valid: $\left\langle\epsilon_{u}\right\rangle^{v, t} \sim \phi v U_{r m s}^{2} / l^{2}$. This corresponds to the $\infty$ regime of classical Rayleigh-Bénard convection (without porous media) introduced by Grossmann \& Lohse (2001) for large $S c$ and small $R a_{f}$. A good agreement between predictions obtained using (4.3) and our DNS results indicates the significance of macroscopic diffusion in momentum transport. 


\section{Macroscopic two-length-scale model for natural convection}

\section{Macroscopic modelling results}

Since the leading-order terms of $D a$ for diffusion are accounted for in the TLSD model, this model can be used in principle to calculate cases characterized by small Darcy numbers. In this section, we test whether and how the model results approach the DNS results as $D a \rightarrow 0$. In addition, we investigate the range of parameters for the validity of the TLSD model.

\subsection{Sherwood number}

Figure 9 shows the relationship between the Sherwood number and the Rayleigh number when $H / s$ is 20 and Rayleigh numbers are up to 20000 . The results of our macroscopic model are compared with the correlation obtained from the DNS results (see Gasow et al. 2020) as well as the DOB results. In the DNS, $S h$ depends on $s / d$ or $\phi$ for $R a>2000$. The value of $S h$ increases as $s / d$ or $\phi$ decreases, while the difference becomes larger as $R a$ increases. In the large-Rayleigh-number regime $(R a \geq 5000)$, the $S h=f(R a)$ scaling changes from a linear scaling $S h \sim R a$ for $s / d=1.25(\phi=0.36)$ to a nonlinear scaling $S h \sim R a^{0.8}$ for $s / d=1.5(\phi=0.56)$, see figure $9(a)$. These characteristics are not captured in DOB simulations but are well reproduced in our macroscopic model simulations.

For the current small $\mathrm{H} / \mathrm{s}$ (or $\mathrm{Da}$ ) value, both DNS and model results show that the Sherwood number increases as the Schmidt number is increased from 1 to 250. Similar to $S c=1$, the scaling for $S c=250$ also changes from linear $(S h \sim R a)$ for $s / d=1.25(\phi=$ $0.36)$ to nonlinear $\left(S h \sim R a^{0.8}\right)$ for $s / d=1.5(\phi=0.56)$. This behaviour is reproduced in our macroscopic model solution, whereas the effect of the Schmidt number is not accounted for in the DOB equations.

We neglected the high-order terms with respect to $D a$ when we proposed the TLSD model. However, since the leading-order term with respect to $D a$ is kept in the momentum equation (2.11), the effect of $D a$ on natural convection can still be accounted for when its value is small enough. The relationship between the Sherwood number and the Darcy number for $R a=20000, S c=1$ or 250 , and $s / d=1.5$ or $1.25(\phi=0.56$ or 0.36$)$ is shown in figure 10. The macroscopic model results are compared with the DNS results as well as with the DOB results. Recall that $a_{v}^{*}$ is only related to the pore-scale geometry and is independent of the flow conditions and the Darcy number. The macroscopic model simulations are in good agreement with the DNS for $D a \leq 2 \times 10^{-6}$ and for different Schmidt numbers. By contrast, the DOB results are independent of the Darcy and Schmidt numbers. The DOB simulations overpredict the Sherwood number for $s / d=1.5(\phi=$ $0.56)$ and underpredict $S h$ for $s / d=1.25(\phi=0.36)$.

\subsection{Species concentration and velocity statistics}

The vertical profiles of the temporally and horizontally averaged macroscopic quantities (time- and line-averaged species concentration $\langle\overline{\hat{c}}\rangle^{x 1}$, species concentration fluctuation $\left\langle\hat{c}^{r m s}\right\rangle^{x 1}$, and velocity fluctuations $\left\langle\hat{u}_{1}^{r m s}\right\rangle^{x 1}$ and $\left.\left\langle\hat{u}_{2}^{r m s}\right\rangle^{x 1}\right)$ for $s / d=1.5(\phi=0.56)$ and $S c=1$ are shown in figure 11. The Rayleigh numbers are 5000 and 20000 . The results of our macroscopic TLSD model are compared with the DNS results as well as the DOB results. It is evident that our macroscopic TLSD modelling results for $\langle\overline{\hat{c}}\rangle^{x 1},\left\langle\hat{u}_{1}^{r m s}\right\rangle^{x 1}$ and $\left\langle\hat{u}_{2}^{r m s}\right\rangle^{x 1}$ are more accurate than the DOB results in the first REV next to the wall. The DNS results show that all statistical results can be well scaled by the pore size $s$ and that the influence of the bounding walls is limited to within the first three REVs next to the 

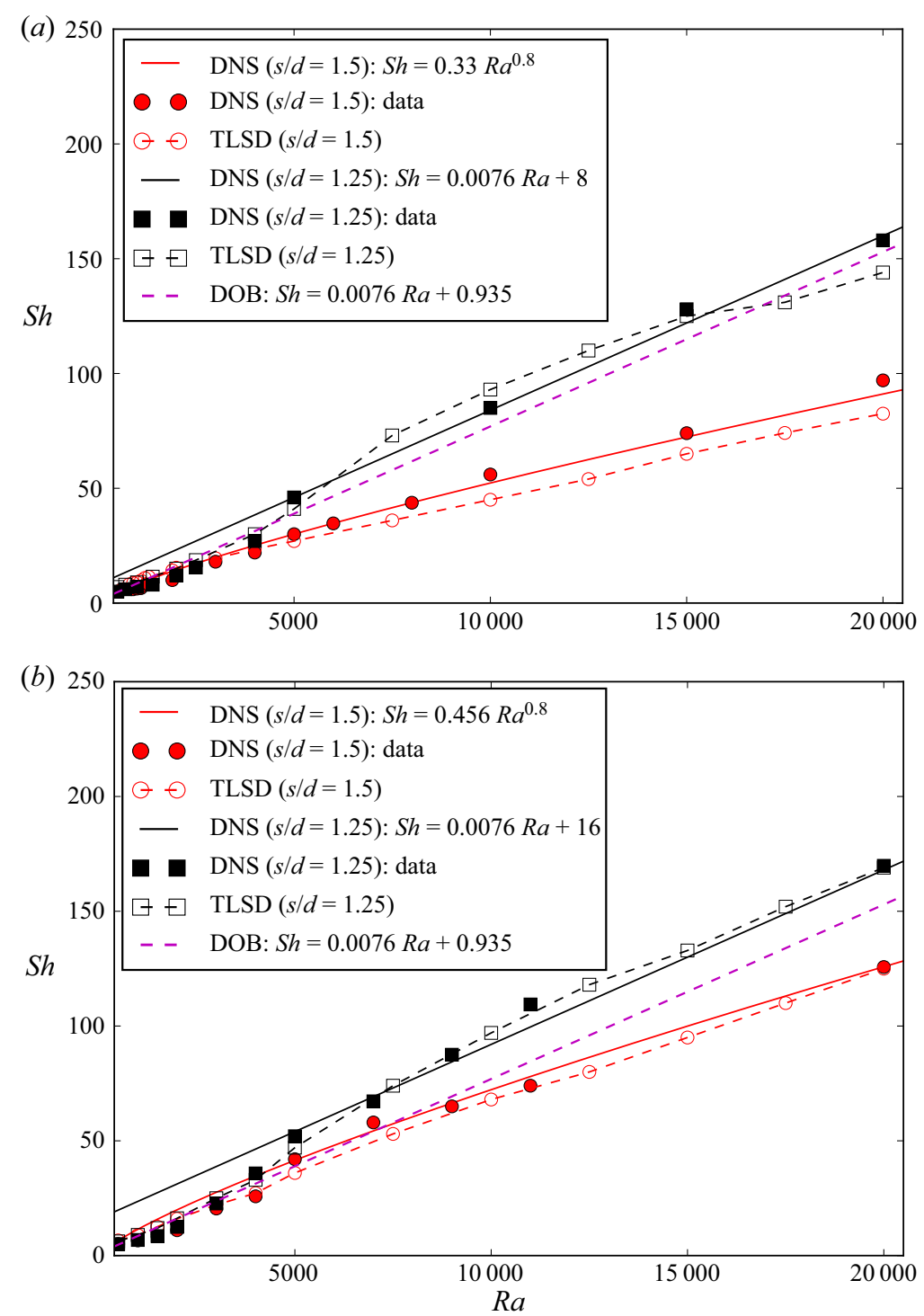

Figure 9. Sherwood number versus the Rayleigh number with $R a$ in the range 500-20 000 and $H / s=20$ for three values of the Darcy number: $D a=8.8 \times 10^{-6}(s / d=1.5), D a=5.4 \times 10^{-6}(s / d=1.4)$ and $D a=$ $1.8 \times 10^{-6}(s / d=1.25)$, with $(a) S c=1$ and $(b) S c=250$.

wall (Gasow et al. 2020). Thus, the boundary layer thickness is determined by the pore size $s$, instead of the Rayleigh number, as suggested in Huppert \& Neufeld (2014). These features are all well captured in the macroscopic simulation.

The same statistical quantities are shown in figure 12 for $S c=250$. It can be seen that these statistical quantities are only marginally changed when a much higher Schmidt number is used in the simulation. Similar to the results for $S c=1$, the macroscopic modelling results are also in good agreement with the DNS results. The macroscopic TLSD model simulation predicts higher mass concentration $\langle\overline{\hat{c}}\rangle^{x 1}$ in the first REV next to the wall and higher transverse velocity fluctuation $\left\langle\hat{u}_{2}^{r m s}\right\rangle^{x 1}$. One possible reason for this discrepancy is that the neglected high-order terms with respect to $D a$ may lead to 

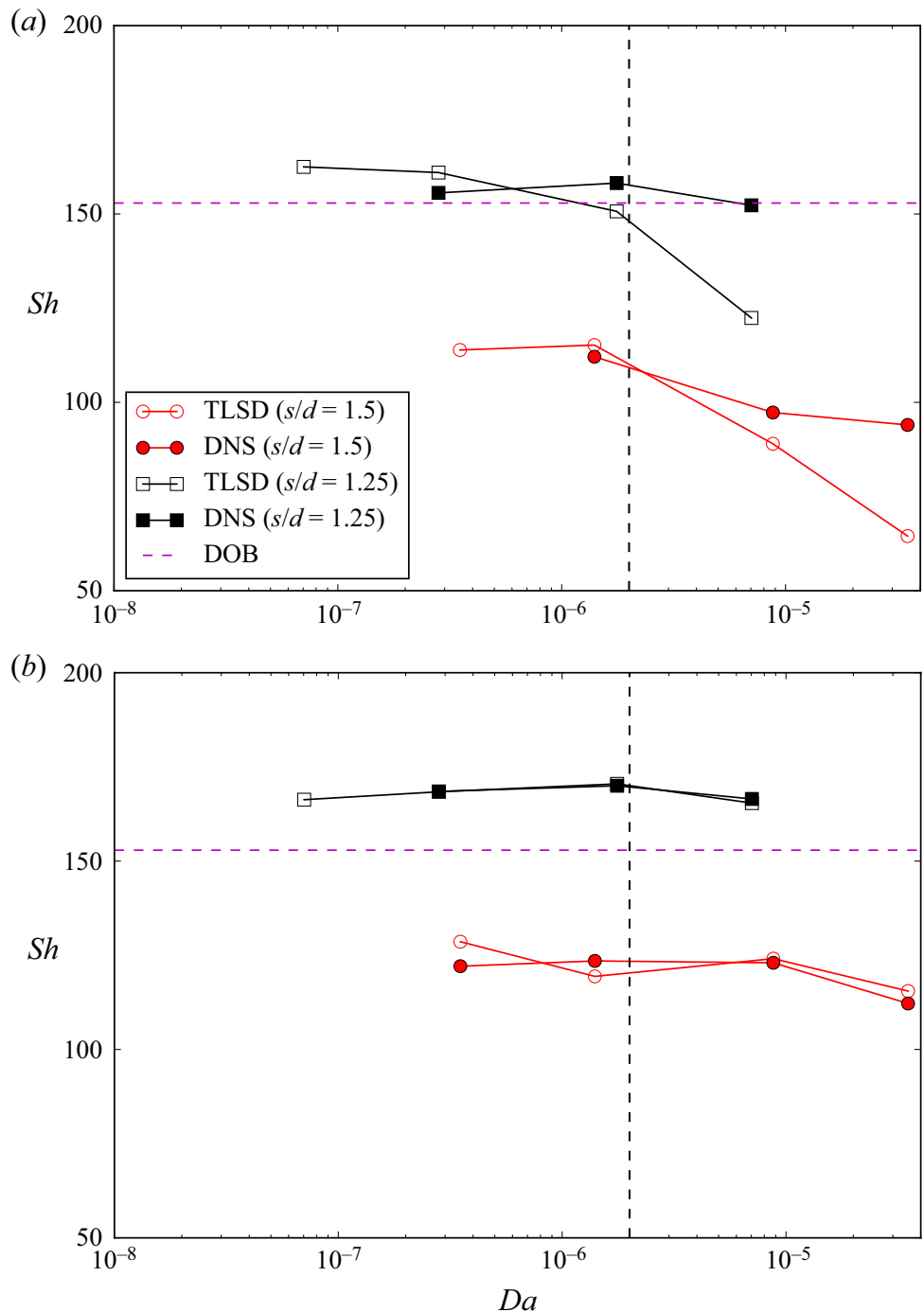

Figure 10. Sherwood number versus the Darcy number for $s / d=1.5 \quad(\phi=0.56)$ with $H / s=$ $10,20,50,100$ and $s / d=1.25(\phi=0.36)$ with $H / s=10,20,50, R a=20000$, for $(a) S c=1$ and $(b)$ $S c=250$.

modelling errors in the boundary layer region. The TLSD model accuracy can be further improved by decomposing the flow domain into a boundary layer region and a central region, so the modelling in the boundary layer region can be improved. However, this would make the model more complicated and difficult to apply. This modelling approach is not adopted in our study to achieve a compromise between the accuracy and simplicity of the macroscopic model.

\subsection{Transient macroscopic fields}

To validate the results of our macroscopic TLSD model, we first compare the transient flow fields obtained from macroscopic simulations of (2.10)-(2.12) with those obtained from the DNS results discussed in the previous section and the DOB simulations reported 

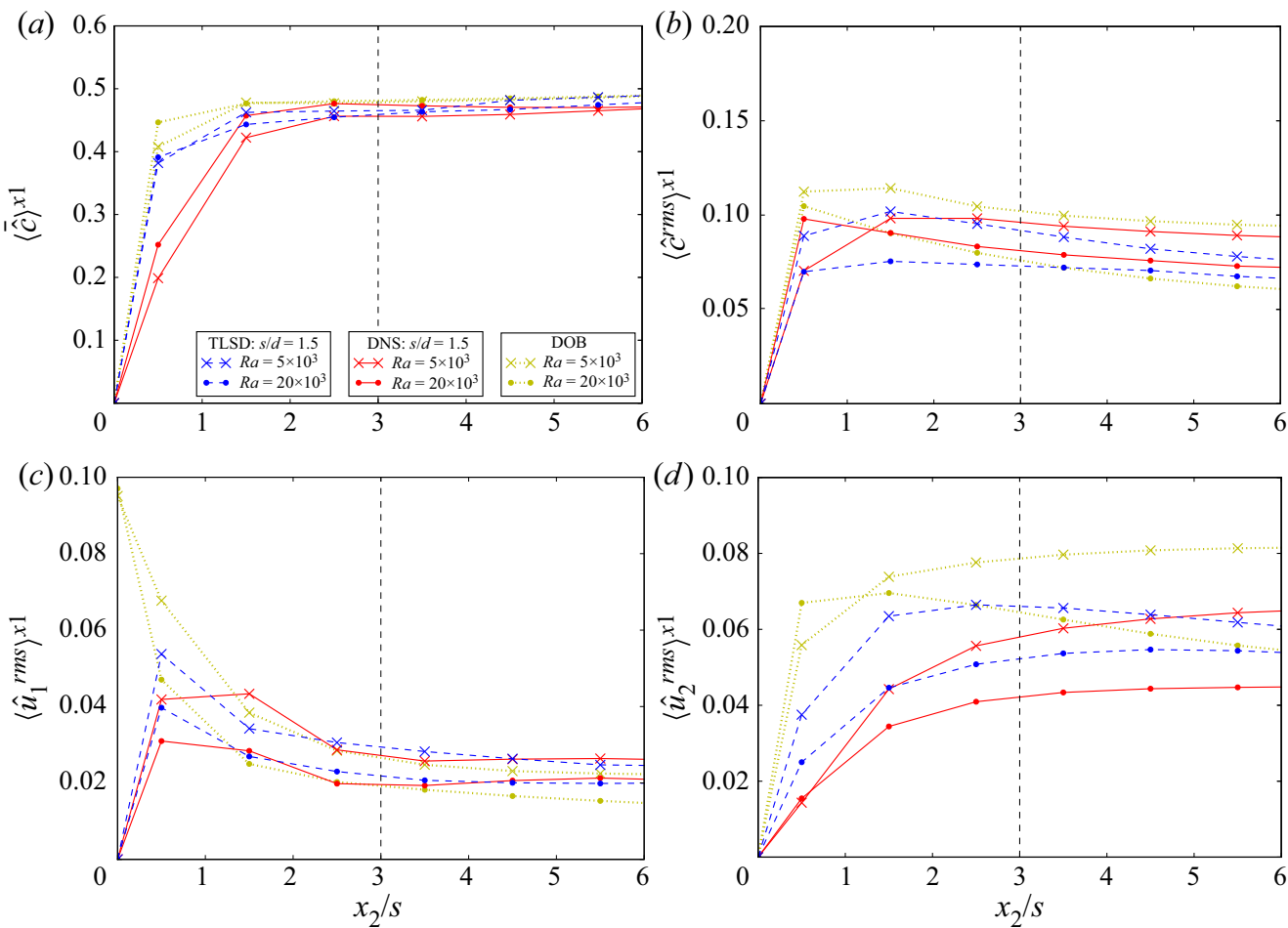

Figure 11. The vertical profiles of the temporally and horizontally averaged macroscopic quantities for $s / d=$ $1.5(\phi=0.56), H / s=20\left(D a=8.8 \times 10^{-6}\right)$ and $S c=1$. The Rayleigh number $R a$ is varied. The distance from the wall is normalized by the pore size $s$. (a) Time- and line-averaged species concentration $\langle\overline{\hat{c}}\rangle^{x 1} ;(b)$ r.m.s. of the species concentration fluctuation $\left\langle\hat{c}^{r m s}\right\rangle^{x 1} ;(c)$ streamwise velocity fluctuation $\left\langle\hat{u}_{1}^{r m s}\right\rangle^{x 1} ;$ and $(d)$ wall-normal velocity fluctuation $\left\langle\hat{u}_{2}^{r m s}\right\rangle^{x 1}$.

in Gasow et al. (2020). For this purpose, the velocity field and the species concentration obtained with the macroscopic simulations and the DNS were volume-averaged (over each REV).

The distribution of the instantaneous $R e_{K}=\left(|\boldsymbol{u}| K^{1 / 2}\right) / v$ for $R a=20000, s / d=$ $1.5(\phi=0.56), H / s=100$ and $S c=250$ is shown in figure 13. The macroscopic TLSD solution (figure 13c) is qualitatively similar to the DNS solution (figure 13a) and DOB solution (figure 13b). Both the DNS solution and macroscopic solutions indicate that the local Reynolds number is $\operatorname{Re}_{K}<4 \times 10^{-3}$. This shows that the studied parameter range is well in the Darcy regime $\left(\operatorname{Re}_{K}<1\right)$, hence the Forchheimer term in the momentum equation can be safely neglected. Despite the laminar flow in the pore scale, the macroscopic flow field is transient and chaotic. However, the strong spatial variation of the velocity field obtained from the DNS is captured neither in TLSD nor in the DOB simulations.

Snapshots of the instantaneous species concentrations for $H / s=100, s / d=1.5(\phi=$ 0.56 ), $R a=20000$ and $S c=250$ are shown in figure 14. The DNS solution (figure $14 a$ ), TLSD solution (figure 14c) and the DOB solution (figure 14b) all exhibit large mega-plume structures in the internal region and small proto-plumes in the boundary layers. They occur due to the rising of a fluid with low species concentration and the sinking of a fluid with 

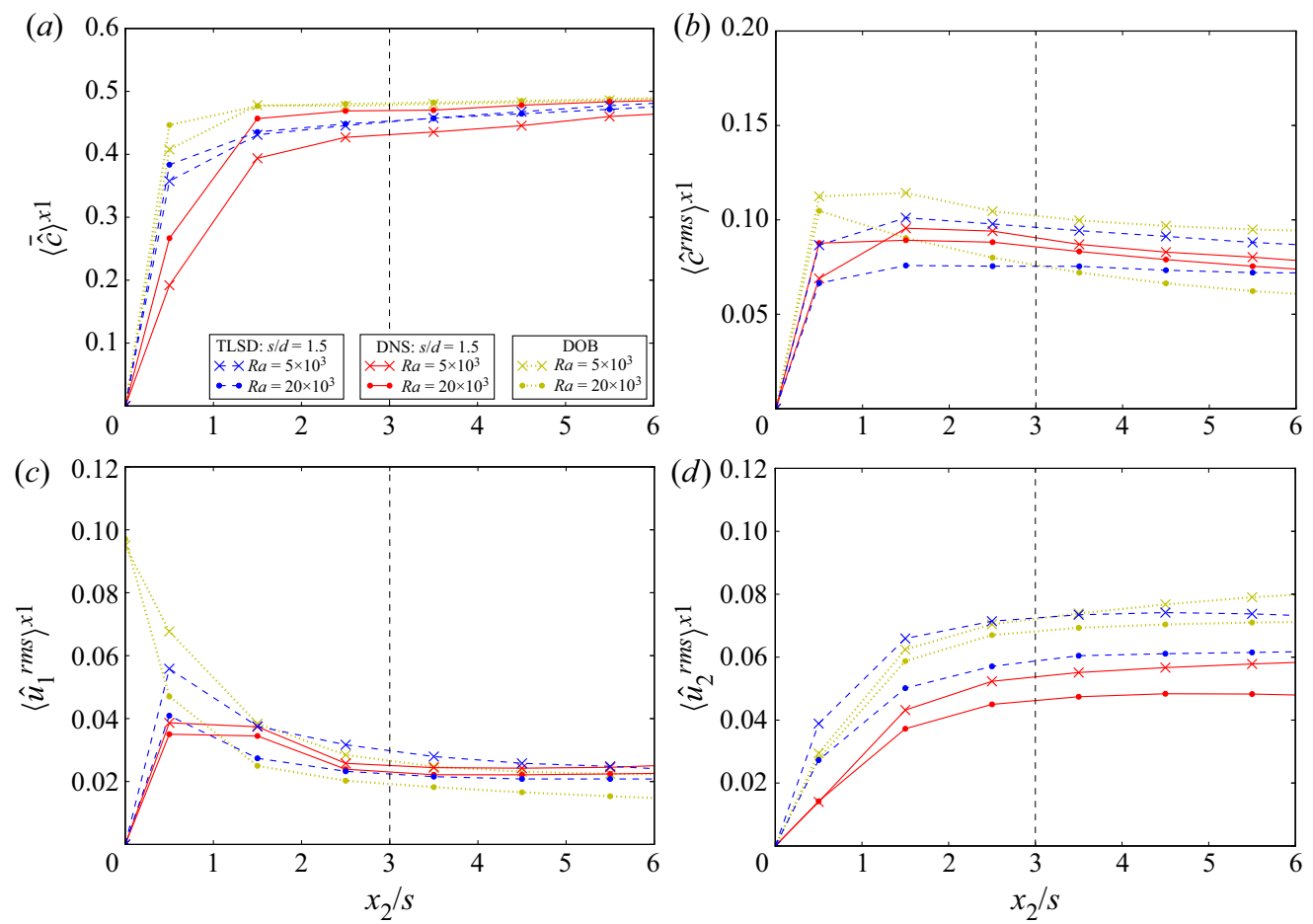

Figure 12. The vertical profiles of the temporally and horizontally averaged macroscopic quantities for $s / d=$ $1.5(\phi=0.56), H / s=20\left(D a=8.8 \times 10^{-6}\right)$ and $S c=250$. The Rayleigh number $R a$ is varied. The distance from the wall is normalized by the pore size $s$. (a) Time- and line-averaged species concentration $\langle\overline{\hat{c}}\rangle^{x 1} ;(b)$ r.m.s. of the species concentration fluctuation $\left\langle\hat{c}^{r m s}\right\rangle^{x 1} ;(c)$ streamwise velocity fluctuation $\left\langle\hat{u}_{1}^{r m s}\right\rangle^{x 1}$; and $(d)$ wall-normal velocity fluctuation $\left\langle\hat{u}_{2}^{r m s}\right\rangle^{x 1}$.

high species concentration, forming the instabilities in the boundary layer region (Hewitt et al. 2012; Kränzien \& Jin 2019).

While the macroscopic TLSD model and DOB solution exhibit relatively regular mega-plumes, in the DNS solution the mega-plumes are more irregular and chaotic. A possible reason is that the Darcy number in our simulation is still not small enough, while the TLSD model is proposed for problems with low Darcy numbers. The transient flow field from macroscopic simulation converges slower than the Sherwood number and other statistical results with decreasing $D a$.

The DNS study reported in Gasow et al. (2020) shows that the number of mega-plumes increases with the decrease of $D a$. Figure 15 shows the time-averaged fast Fourier transform (FFT) of the dimensionless mass concentration $\hat{c}$ along the centreline at $x_{2}=$ $H / 2$. The peak wavenumber calculated from the TLSD simulation is still higher than the DNS result, but it is lower than the DOB result. Figure 16 shows that the peak wavenumber from DNS approaches the TLSD or DOB results as the Darcy number approaches 0 . However, DNS of natural convection with smaller Darcy numbers are still needed to confirm that the peak wavenumber from DNS will not exceed the TLSD results and approach the DOB results.

The three-dimensional DOB simulations by Pirozzoli et al. (2021) revealed the supercells at the boundary, which are the footprints of mega-plumes dominating the interior part of the flow. They suggest that these supercells might lead to the nonlinear 

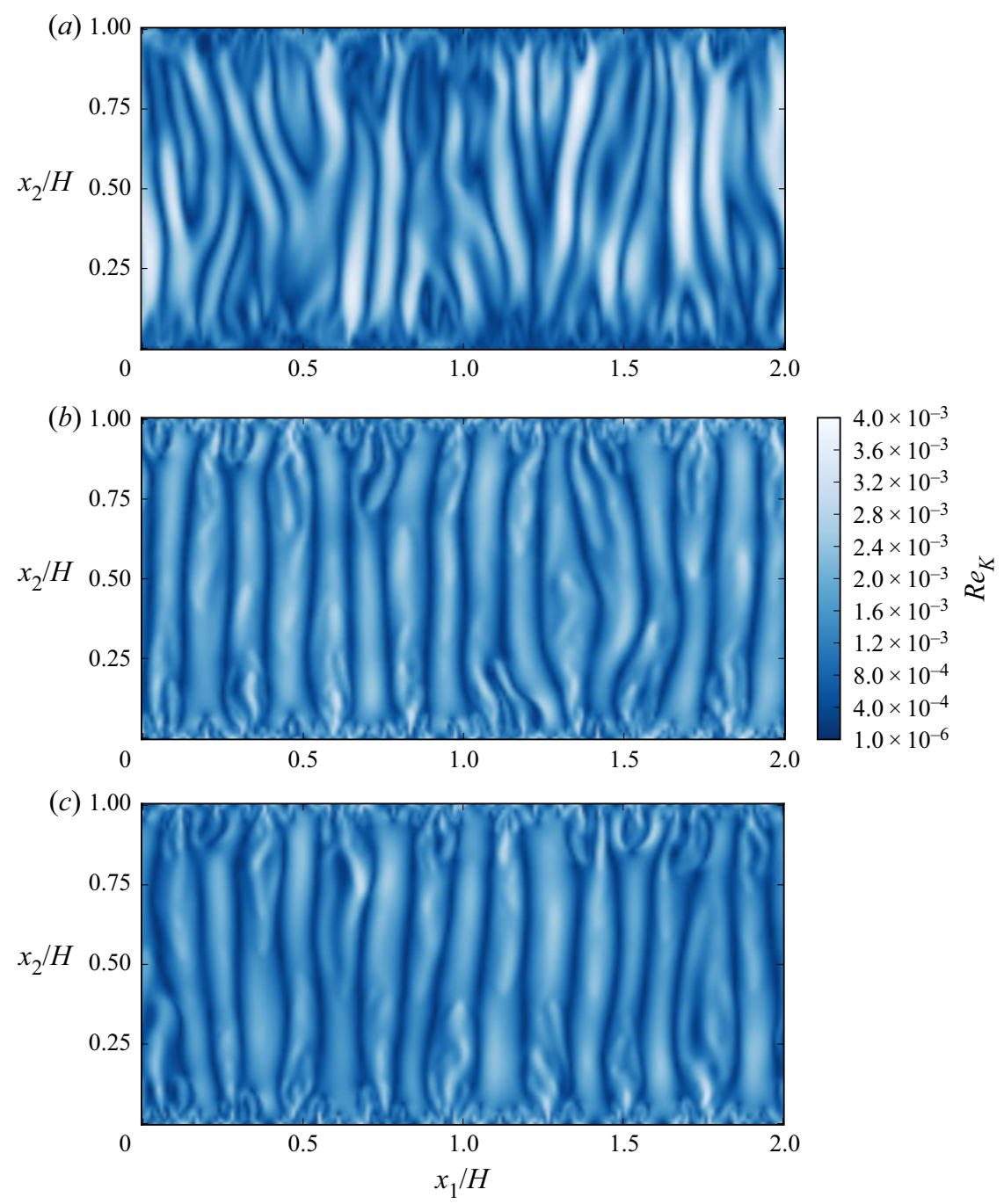

Figure 13. Instantaneous volume-averaged Reynolds number $R e_{K}, H / s=100\left(D a=3.5 \times 10^{-7}\right)$, $s / d=1.5(\phi=0.56), R a=20000$ and $S c=250:(a)$ DNS, (b) DOB and (c) TLSD.

scaling of $S h(R a)$ in the ultimate regime of high $R a$ numbers. Future work needs to investigate whether these supercells will be also captured by three-dimensional TLSD simulations. Elucidating how macroscopic diffusion affects the plume structures is also a subject of future investigation.

\section{Conclusions}

The DNS results of Gasow et al. (2020) (extended in this study) show that the pore-scale geometry also has significant effects on natural convection in porous media, in particular, the boundary layer thickness is determined by the pore size instead of the Rayleigh number. Based on this, we have proposed the following TLSD model: we assume that pore-scale structures affect the momentum transport through macroscopic diffusion. The macroscopic diffusion is of the same order with respect to the Darcy number as the Darcy 

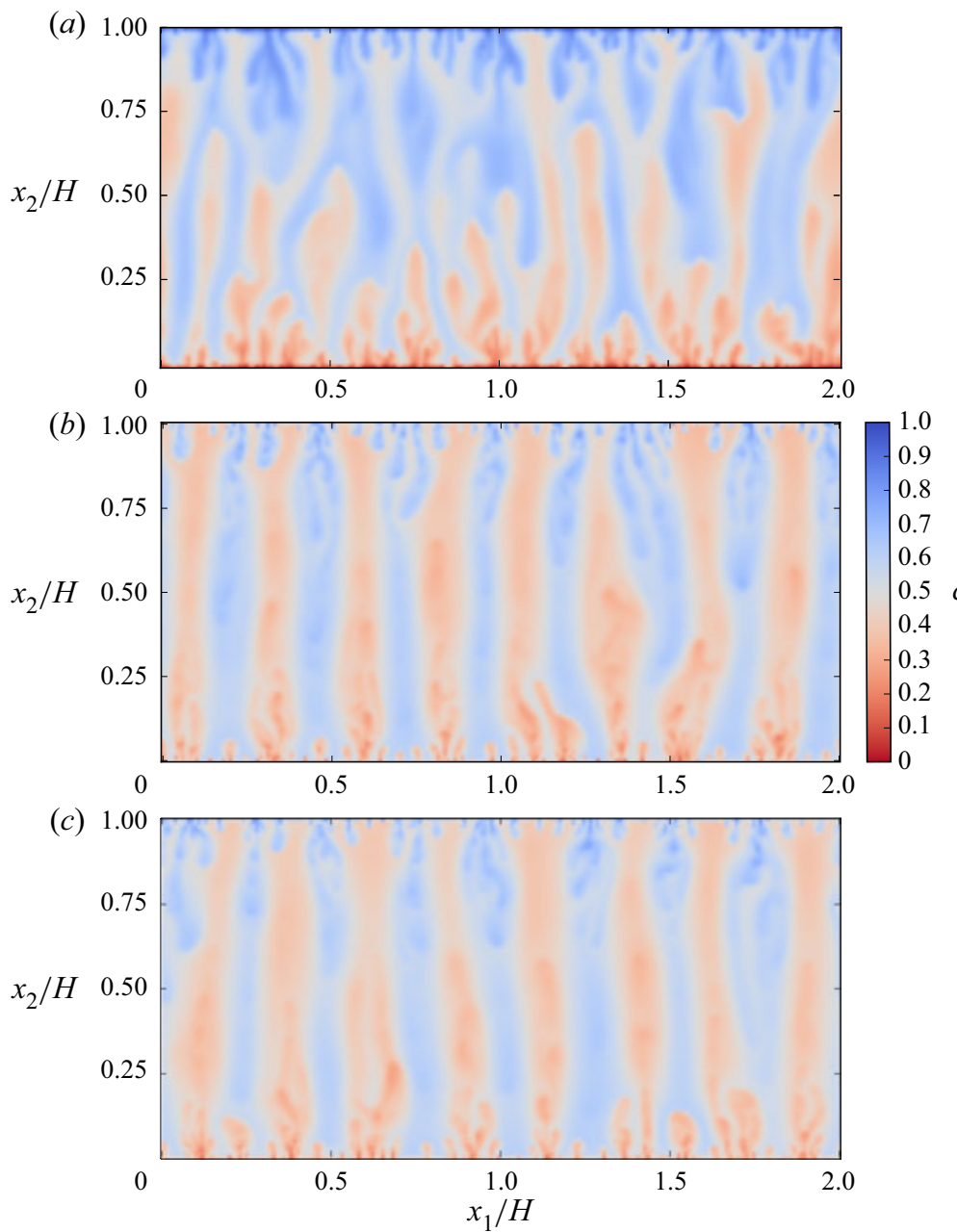

Figure 14. Instantaneous volume-averaged species concentration $\hat{c}, H / s=100\left(D a=3.5 \times 10^{-7}\right)$, $s / d=1.5(\phi=0.56), R a=20000$ and $S c=250:(a)$ DNS, $(b)$ DOB and $(c)$ TLSD.

drag and the buoyancy force; thus, it cannot be neglected even if the Darcy number is small. It is determined by two length scales: the pore size characterized by $\sqrt{K}$ and the distance between the lower and upper boundaries $H$.

The DNS results show that the loss of the macroscopic kinetic energy is mainly due to microscopic diffusion and the pressure gradient. The loss captured in Darcy's law is only part of the overall loss, even if the superficial velocity is accurately calculated in the DOB equation. The macroscopic diffusion term added here to the momentum equation accounts for the additional loss of the macroscopic kinetic energy. Our DNS results also show that the Sherwood number is almost independent of the Darcy number when the Darcy number is small enough. Thus, the diffusion term is of the same order of the Darcy number $\left(D a=K / H^{2}\right)$ as the buoyancy force term and the Darcy term.

A new macroscopic model for simulating natural convection in porous media is developed based on the TLSD assumption. The results of our model are validated extensively by comparison with the DNS as well as the DOB results. The comparison 


\section{S. Gasow, A.V. Kuznetsov, M. Avila and Y. Jin}

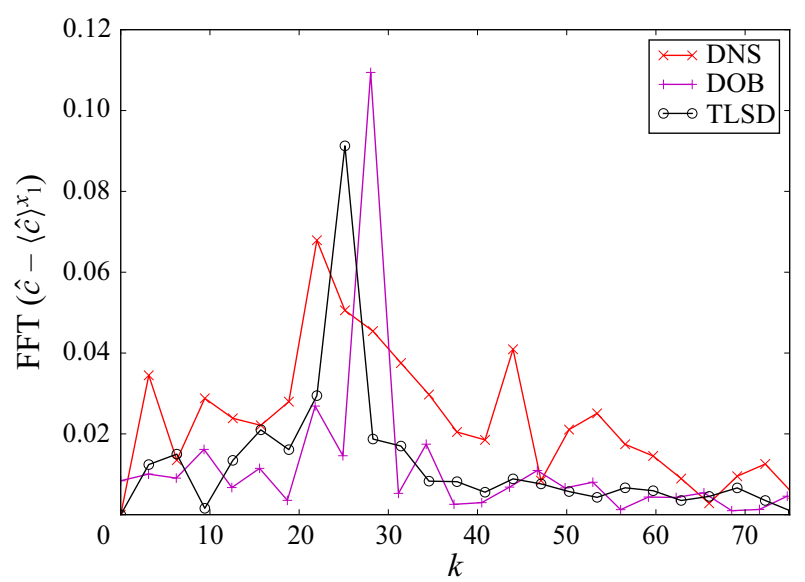

Figure 15. Average spectra of the dimensionless mass concentration, $\hat{c}$, of the DNS, DOB and TLSD results at mid-height $x_{2}=H / 2, H / s=100\left(D a=3.5 \times 10^{-7}\right), s / d=1.5(\phi=0.56), R a=20000$ and $S c=250$.

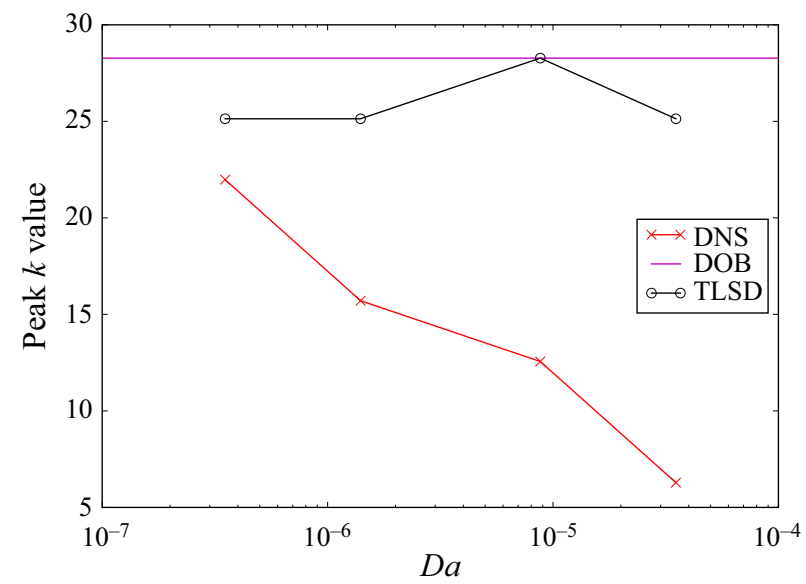

Figure 16. Peak wavenumber $k$ for the mega-plumes of the DNS, DOB and TLSD results for different Darcy numbers with $s / d=1.5(\phi=0.56), R a=20000$ and $S c=250$.

shows that the new macroscopic model performs well as long as $D a \leq 2 \times 10^{-6}$. Simulations of the model predict a much more accurate Sherwood number, r.m.s. mass concentration and r.m.s. velocity than simulations that employ the DOB equations. They also predict the structures of mega-plumes and proto-plumes with reasonable accuracy. In particular, the new model results show that the $S h=f(R a)$ scaling changes from a linear scaling to a nonlinear scaling as the porosity increases. If the Rayleigh number and Darcy number are fixed, the Sherwood number increases with the increase of the Schmidt number and the decrease of the porosity. These trends agree with the DNS results, whereas they cannot be captured by the DOB simulations. We expect that these trends, as well as the TLSD assumption, also apply to three-dimensional flows. However, how macroscopic diffusion affects the plume structures remains an open question.

Some discrepancies between the new macroscopic modelling results and the DNS results can be found in the boundary layer. The new macroscopic model over predicts the mean mass concentration in the first REV next to the wall. This error may be reduced if the 


\section{Macroscopic two-length-scale model for natural convection}

higher-order terms with respect to $D a$, e.g. the mass dispersion, are considered. However, the current macroscopic model appears preferable due to its simplicity.

This work is the first step towards modelling fundamental issues arising at the pore scale in $\mathrm{CO}_{2}$ sequestration processes. However, it should be noted that a real $\mathrm{CO}_{2}$ sequestration process is much more complicated. It has been extensively investigated by numerical modelling in the last two decades (Weir, White \& Kissling 1995, 1996; Lindeberg \& Wessel-Berg 1997; Hassanzadeh, Pooladi-Darvish \& Keith 2005, 2007; Bickle et al. 2007; Pruess \& Zhang 2008; Chen et al. 2018). It is characterized by three-dimensional, inherently transient multiphase flow with much more complicated pore-scale geometries and much lower Darcy numbers than those studied in this research (Michael et al. 2010; Riley 2010; Huppert \& Neufeld 2014).

Funding. The authors gratefully acknowledge the support of this study by the DFG (Deutsche Forschungsgemeinschaft, 408356608) and the HLRN (North-German Supercomputing Alliance). A.V.K. acknowledges with gratitude the support of the National Science Foundation (award CBET-2042834) and the Alexander von Humboldt Foundation through the Humboldt Research Award.

Declaration of interests. The authors report no conflict of interest.

Author ORCIDs.

(1) Andrey V. Kuznetsov https://orcid.org/0000-0002-2692-6907;

(1) Marc Avila https://orcid.org/0000-0001-5988-1090;

(ㄷ) Yan Jin https://orcid.org/0000-0002-2297-6827.

\section{Appendix A. Momentum dispersion and species concentration dispersion}

Breugem, Boersma \& Uittenbogaard (2006) argued that momentum dispersion has negligible effects on convection in porous media. This agrees with the study by Rao et al. (2020), who showed numerically that momentum dispersion should be accounted for only when the local Reynolds number $\operatorname{Re}_{K} \gg 1$. Hence, the momentum dispersion is neglected here as well.

The effects of mass dispersion (or thermal dispersion for heat transfer problems) have been extensively studied in recent years, as discussed in the Introduction (Liang et al. 2018; Wen et al. 2018b; Alomar 2019; Fahs et al. 2020). The dispersion term in (2.7) is often modelled using a Fickian dispersion tensor, first introduced by Bear (1961) and expressed as

$$
\phi\left\langle{ }^{i} u_{i}{ }^{i} c\right\rangle^{i}=D_{i j} \frac{\partial \breve{c}}{\partial \breve{x}_{j}},
$$

where the dispersion tensor $D_{i j}$ is calculated as

$$
D_{i j}=\left(\alpha_{l}-\alpha_{t}\right) \breve{u}_{i} \breve{u}_{j} /|\breve{\boldsymbol{u}}|+\alpha_{t} \delta_{i j}|\breve{\boldsymbol{u}}|,
$$

with $\alpha_{l}$ and $\alpha_{t}$ the longitudinal and transverse diffusivities, respectively. They can be determined from the numerical results for the flow and mass transfer in an REV with a linear concentration gradient in the streamwise or transverse direction; see Nakayama \& Kuwahara (1999) and Pedras \& de Lemos (2008). These studies suggest that $D_{i j}$ has a scaling of the form $D_{i j} \sim P e_{K}^{n}$, where the local Péclet number $P e_{K}$ is defined as

$$
P e_{K}=R e_{K} S c=\frac{|\breve{u}|}{u_{m}} R e_{m} D a^{1 / 2},
$$

where $R e_{K}=|\breve{\boldsymbol{u}}| \sqrt{K} / v$ and $R e_{m}=u_{m} H / v$ are the local and global Reynolds numbers, respectively. Delgado (2007) and Nakayama \& Kuwahara (1999) suggested that the scaling 


\section{S. Gasow, A.V. Kuznetsov, M. Avila and Y. Jin}

coefficient $n$ is between 1 and 2. As a consequence, $D_{i j}$ is expected to be of order between $D a^{1 / 2}$ and $D a$, while $D_{m}$ is of order $D a^{0}$. When the Darcy number is small enough, $\left|D_{i j}\right| \ll D_{m}$. Since we are interested in natural convection with small Darcy numbers, we only retain the leading-order terms with respect to $D a$ for diffusion in (2.7). Thus, due to this theoretical derivation, mass dispersion can also be neglected.

The dispersion at the macroscale (macrodispersivity) suggested by Gelhar et al. (1992), Lallemand-Barres \& Peaudecerf (1978), Neuman (1990) and Liang et al. (2018) is not considered in this study since its effect on the plume scale has not yet been fully elucidated (Zech et al. 2019). Instead, the effect of dispersion is modelled as macroscopic diffusion in the momentum equation. The macroscopic diffusion affects the velocity field and then accounts for the dispersion in the species concentration indirectly.

\section{REFERENCES}

Alomar, O.R. 2019 Analysis of variable porosity, thermal dispersion, and local thermal non-equilibrium on two-phase flow inside porous media. Appl. Therm. Engng 154, 263-283.

Arts, R.J., Chadwick, A., Eiken, O., Thibeau, S. \& Nooner, S. 2008 Ten years' experience of monitoring $\mathrm{CO}_{2}$ injection in the Utsira Sand at Sleipner, offshore Norway. First Break 26 (1), 65-72.

Backhaus, S., TURITSYN, K. \& ECKE, R.E. 2011 Convective instability and mass transport of diffusion layers in a Hele-Shaw geometry. Phys. Rev. Lett. 106, 104501.

Baehr, H.D. \& StePhan, K. 2006 Heat and Mass Transfer, 2nd edn. Springer.

Basbug, B. \& Gumrah, F. 2009 Parametric study of carbon dioxide sequestration in deep saline aquifers. Heat Transfer Engng 31, 255-272.

BEAR, J. 1961 On the tensor form of dispersion in porous media. J. Geophys. Res. 66 (4), 1185-1197.

Bickle, M., Chadwick, A., Huppert, H.E., Hallworth, M. \& Lyle, S. 2007 Modelling carbon dioxide accumulation at sleipner: implications for underground carbon storage. Earth Planet. Sci. Lett. $255(1-2), 164-176$.

Böttcher, N., Watanabe, N., Görke, U.-J. \& Kolditz, O. 2016 Geoenergy Modeling I. Springer.

Breugem, W.P., Boersma, B.J. \& UitTEnbogaARD, R.E. 2006 The influence of wall permeability on turbulent channel flow. J. Fluid Mech. 562, 35-72.

BRINKMAN, H.C. 1949 A calculation of the viscous force exerted by a flowing fluid on a dense swarm of particles. Flow Turbul. Combust. 1 (1), 27-34.

Chen, B., Harp, D.R., Lin, Y., Keating, E.H. \& Pawar, R.J. 2018 Geologic $\mathrm{CO}_{2}$ sequestration monitoring design: a machine learning and uncertainty quantification based approach. Appl. Energy 225, 332-345.

DARCY, H.P.G. 1856 Les Fontaines publiques de la ville de Dijon. Exposition et application des principes à suivre et des formules à employer dans les questions de distribution d'eau, etc. V. Dalamont.

DAS, D., BISWAL, P., RoY, M. \& BASAK, T. 2016 Role of the importance of 'Forchheimer term' for visualization of natural convection in porous enclosures of various shapes. Intl J. Heat Mass Transfer 97, 1044-1068.

De Lemos, M.J.S. 2012 Turbulence in Porous Media, Modeling and Applications, 2nd edn. Elsevier.

De Paoli, M., Alipour, M. \& Soldati, A. 2020 How non-Darcy effects influence scaling laws in Hele-Shaw convection experiments. J. Fluid Mech. 892, A41.

De PAOli, M., ZonTA, F. \& SoldATI, A. 2016 Influence of anisotropic permeability on convection in porous media: implications for geological CO2 sequestration. Phys. Fluids 28 (5), 056601.

Delgado, J.M.P.Q. 2007 Longitudinal and transverse dispersion in porous media. Chem. Engng Res. Des. 85 (9), 1245-1252.

Fahs, M., Graf, T., Tran, T.V., Ataie-Ashtiani, B., Simmons, C.T. \& Younes, A. 2020 Study of the effect of thermal dispersion on internal natural convection in porous media using Fourier series. Trans. Porous Med. 131 (2), 537-568.

Faisal, T.F., Chevalier, S., Bernabe, Y., Juanes, R. \& SAssi, M. 2015 Quantitative and qualitative study of density driven $\mathrm{CO}_{2}$ mass transfer in a vertical Hele-Shaw cell. Intl J. Heat Mass Transfer 81, 901-914.

Gasow, S., Lin, Z., Zhang, H.C., Kuznetsov, A.V., Avila, M. \& Jin, Y. 2020 Effects of pore scale on the macroscopic properties of natural convection in porous media. J. Fluid Mech. 891, A25.

Gelhar, L.W., Welty, C. \& RehfeldT, K.R. 1992 A critical review of data on field-scale dispersion in aquifers. Water Resour. Res. 28, 1955-1974. 


\section{Macroscopic two-length-scale model for natural convection}

Ghesmat, K., Hassanzadeh, H. \& Abedi, J. 2011 The effect of anisotropic dispersion on the convective mixing in long-term $\mathrm{CO}_{2}$ storage in saline aquifers. AIChE J. 57, 561-570.

Ghoreishi-Madiseh, S.A., Hassani, F.P., Mohammadian, A. \& Radziszewski, P.H. 2013 A transient natural convection heat transfer model for geothermal borehole heat exchangers. J. Renew. Sustain. Energy 5 (4), 043104.

Grossmann, S. \& LohSE, D. 2000 Scaling in thermal convection: a unifying theory. J. Fluid Mech. 407, $27-56$.

Grossmann, S. \& Lohse, D. 2001 Thermal convection for large Prandtl numbers. Phys. Rev. Lett. 86 (15), 3316-3319.

Grossmann, S. \& LohsE, D. 2004 Fluctuations in turbulent Rayleigh-Bénard convection: the role of plumes. Phys. Fluids 16 (12), 4462-4472.

Hassanzadeh, H., Pooladi-Darvish, M. \& Keith, D.W. 2005 Modelling of convective mixing in $\mathrm{CO}_{2}$ storage. J. Can. Petrol. Technol. 44 (10), 43-51.

Hassanzadeh, H., Pooladi-Darvish, M. \& Keith, D.W. 2007 Scaling behavior of convective mixing, with application to geological storage of $\mathrm{CO}_{2}$. AIChE J. 53 (5), 1121-1131.

HERWIG, H. 2013 Wärmeübertragung A-Z: systematische und ausführliche Erläuterungen wichtiger Größen und Konzepte, 1 st edn. Springer-Verlag.

HeRwig, H. \& Moschallski, A. 2009 Wärmeübertragung, 2nd edn. Vieweg+ Teubner.

HewitT, D.R. 2020 Vigorous convection in porous media. Proc. R. Soc. A 476 (2239), 20200111.

Hewitt, D.R., Neufeld, J.A. \& Lister, J.R. 2012 Ultimate regime of high Rayleigh number convection in a porous medium. Phys. Rev. Lett. 108, 224503.

Hewitt, D.R., Neufeld, J.A. \& Lister, J.R. 2013 Convective shutdown in a porous medium at high Rayleigh number. J. Fluid Mech. 719, 551-586.

Hewitt, R., Neufeld, J.A. \& Lister, J.R. 2014 High Rayleigh number convection in a three-dimensional porous medium. J. Fluid Mech. 748, 879-895.

Heyde, M.V.D. \& Schmitz, G. 2017 Particle-resolved CFD-simulations of thermal energy storage in rock beds. In 11th International Renewable Energy Storage Conference, IRES 2017, 14-16 March 2017. Düsseldorf, Germany.

HIDALGO, J.J. \& CARRERA, J. 2009 Effect of dispersion on the onset of convection during $\mathrm{CO}_{2}$ sequestration. J. Fluid Mech. 640, 441-452.

Huppert, H.E. \& Neufeld, J.A. 2014 The fluid mechanics of carbon dioxide sequestration. Annu. Rev. Fluid Mech. 46, 255-272.

JAVAHERI, M., ABEDI, J. \& HASSANZADEH, H. 2010 Linear stability analysis of double- diffusive convection in porous media, with application to geological storage of $\mathrm{CO}_{2}$. Trans. Porous Med. 84 (2), 441-456.

JIN, Y. \& KUZNETSOV, A.V. 2017 Turbulence modeling for flows in wall bounded porous media: an analysis based on direct numerical simulations. Phys. Fluids 29, 045102.

Jin, Y., Uth, M.F., KuZnetsov, A.V. \& Herwig, H. 2015 Numerical investigation of the possibility of macroscopic turbulence in porous media: a DNS study. J. Fluid Mech. 766, 76-103.

JOUYBARI, N.F., LUNDSTRÖM, T.S. \& HELLSTRÖM, J.G.I. 2020 Investigation of thermal dispersion and intra-pore turbulent heat flux in porous media. Intl J. Heat Fluid Flow 81, 108523.

KeEne, D.J. \& Goldstein, R.J. 2015 Thermal convection in porous media at high Rayleigh numbers. J. Heat Transfer 137 (3), 034503.

KneAfSeY, T.J. \& PRUess, K. 2010 Laboratory flow experiments for visualizing carbon dioxide-induced, density-driven brine convection. Trans. Porous Med. 82 (1), 123-139.

KRÄNZIEN, P.U. \& JIN, Y. 2019 Natural convection in a two-dimensional cell filled with a porous medium: a direct numerical simulation study. Heat Transfer Engng 40, 487-496.

Kunes, J. 2012 Dimensionless Physical Quantities in Science and Engineering. Elsevier.

Letelier, J.A., MujicA, N. \& ORTEgA, J.H. 2019 Perturbative corrections for the scaling of heat transport in a Hele-Shaw geometry and its application to geological vertical fractures. J. Fluid Mech. 864, 746-767.

Liang, Y., Wen, B., Hesse, M. \& DiCarlo, D. 2018 Effect of dispersion on solutal convection in porous media. Geophys. Res. Lett. 45, 9690.

LiNDEBERG, E. \& WeSSEL-BERG, D. 1997 Vertical convection in an aquifer column under a gas cap of $\mathrm{CO}_{2}$. Energy Convers. Manage. 38, 229-234.

Liu, S., Jiang, L., Chong, K.L., Zhu, X., Wan, Z.H., Verzicco, R., Stevens, R.J.A.M., Lohse, D. \& SUN, C. $2020 a$ From Rayleigh-Bénard convection to porous-media convection: how porosity affects heat transfer and flow structure. J. Fluid Mech. 895, A18.

LiU, H., PATIL, P.R. \& NARUSAWA, U. 2007 On Darcy-Brinkman equation: viscous flow between two parallel plates packed with regular square arrays of cylinders. Entropy 9 (3), 118-131. 


\section{S. Gasow, A.V. Kuznetsov, M. Avila and Y. Jin}

LiU, S., ZhAng, Y., ZhAO, J., JiAnG, L. \& Song, Y. $2020 b$ Dispersion characteristics of $\mathrm{CO}_{2}$ enhanced gas recovery over a wide range of temperature and pressure. J. Natural Gas Sci. Engng 73, 103056.

MacMinn, C.W., Neufeld, J.A., Hesse, M.A. \& Huppert, H.E. 2012 Spreading and convective dissolution of carbon dioxide in vertically confined, horizontal aquifers. Water Resour. Res. 48 (11), W11516.

MesquitA, M.S. \& DE Lemos, M.J. 2004 Optimal multigrid solutions of two-dimensional convection-conduction problems. Appl. Maths Comput. 152 (3), 725-742.

Metz, B, Davidson, O. \& De Coninck, H. (Eds.) 2005 Carbon Dioxide Capture and Storage: Special Report of the Intergovernmental Panel on Climate Change. Cambridge University Press.

Michael, K., Arnot, M., Cook, P., Ennis-King, J., Funnell, R., Kaldi, J., Kirste, D. \& PATERSON, L. $2009 \mathrm{CO}_{2}$ storage in saline aquifers I-Current state of scientific knowledge. Energy Procedia 1 (1), 3197-3204.

Michael, K., Golab, A., Shulakova, V., Ennis-King, J., Allinson, G., Sharma, S. \& Aiken, T. 2010 Geological storage of $\mathrm{CO}_{2}$ in saline aquifers - a review of the experience from existing storage operations. Intl J. Greenh. Gas Control 4 (4), 659-667.

Mijic, A., LAforce, T.C. \& Muggeridge, A.H. $2014 \mathrm{CO}_{2}$ injectivity in saline aquifers: the impact of non-Darcy flow, phase miscibility, and gas compressibility. Water Resour. Res. 50, 4163-4185.

Minkowycz, W.J., Sparrow, E.M., Schneider, G.E. \& Pletcher, R.H. 2006 Handbook of Numerical Heat Transfer, 2nd edn. John Wiley \& Sons.

NAKAyAma, A. \& KUWAhaRA, F. 1999 A macroscopic turbulence model for flow in a porous medium. J. Fluids Engng 121 (2), 427-433.

Neufeld, J.A., Hesse, M.A., Riaz, A., Hallworth, M.A., Techelepi, H.A. \& Huppert, H.E. 2010 Convective dissolution of carbon dioxide in saline aquifers. Geophys. Res. Lett. 27, L22404.

NEUMAN, S.P. 1990 Universal scaling of hydraulic conductivities and dispersivities in geologic media. Water Resour. Res. 26 (8), 1749-1758.

NIELD, D.A. 1994 Estimation of an effective Rayleigh number for convection in a vertically inhomogeneous porous medium or clear fluid. Intl J. Heat Fluid Flow 15 (4), 337-340.

Nield, A. \& Bejan, A. 2017 Convection in Porous Media, 5th edn. Springer.

OCHOA-TAPIA, J.A. \& WhitAKER, S. 1995 Momentum transfer at the boundary between a porous medium and a homogeneous fluid-I. Theoretical development. Intl J. Heat Mass Transfer 38 (14), 2635-2646.

ORR, F.M. 2009 Onshore geologic storage of $\mathrm{CO}_{2}$. Science 325 (5948), 1656-1658.

PAMUKCU, Y.Z. \& GumRAH, F. 2009 A numerical simulation study of carbon-dioxide sequestration into a depleted oil reservoir. Heat Transfer Engng 31, 1348-1367.

PedrAS, M.H. \& DE Lemos, M.J. 2008 Thermal dispersion in porous media as a function of the solid-fluid conductivity ratio. Intl J. Heat Mass Transfer 51 (21), 5359-5367.

Pirozzoli, S., De Paoli, M., Zonta, F. \& Soldati, A. 2021 Towards the ultimate regime in Rayleigh-Darcy convection. J. Fluid Mech. 911, R4.

PRUESS, K. \& ZHANG, K. 2008. Numerical modeling studies of the dissolution-diffusion- convection processduring CO2 storage in saline aquifers (No. LBNL-1243E). Lawrence Berkeley National Lab. (LBNL), Berkeley, CA.

RaO, F., KuZnetsov, A.V. \& Jin, Y. 2020 Numerical modeling of momentum dispersion in porous media based on the pore scale prevalence hypothesis. Trans. Porous Med. 133 (2), 271-292.

RILEY, N. 2010 Geological storage of carbon dioxide. Issues Environ. Sci. Technol. 29, 155-178.

Shao, Q., Fahs, M., Younes, A. \& Makradi, A. 2016. A high-accurate solution for Darcy- Brinkman double-diffusive convection in saturated porous media. Numer. Heat Transfer B 69(1), $26-47$.

Singh, H., SAini, R.P. \& SAINI, J.S. 2010 A review on packed bed solar energy storage systems. Renew. Sustain. Energy Rev. 14 (3), 1059-1069.

Starov, V.M. \& Zhdanov, V.G. 2001 Effective viscosity and permeability of porous media. Colloids Surf. A: Physicochem. Engng Aspects 192 (1-3), 363-375.

Torabi, M., Karimi, N., Peterson, G.P. \& Yee, S. 2017 Challenges and progress on the modelling of entropy generation in porous media: a review. Intl J. Heat Mass Transfer 114, 31-46.

Uth, M.F., Jin, Y., KuZnetsov, A.V. \& Herwig, H. 2016 A DNS study on the possibility of macroscopic turbulence in porous media: effects of different solid matrix geometries, solid boundaries, and two porosity scales. Phys. Fluids 28, 065101.

VAFAI, K. (Ed.) 2005 Handbook of Porous Media, 2nd edn. CRC Press.

VALDES-PARADA, F.J., OCHOA-TAPIA, J.A. \& AlvAREZ-RAMIREZ, J. 2007 On the effective viscosity for the Darcy-Brinkman equation. Phys. A: Stat. Mech. Appl. 385 (1), 69-79.

VAsseur, P., WANG, C.H. \& SEN, M. 1989 The Brinkman model for natural convection in a shallow porous cavity with uniform heat. Numer. Heat Transfer A: Appl. 15 (2), 221-242. 


\section{Macroscopic two-length-scale model for natural convection}

Versteeg, H.K. \& Malalasekera, W. 2007 An Introduction to Computational Fluid Dynamics: The Finite Volume Method, 2nd edn. Pearson education.

Wang, L., Nakanishi, Y., Hyodo, A. \& Suekane, T. 2016 Three-dimensional structure of natural convection in a porous medium: effect of dispersion on finger structure. Intl J. Greenh. Gas Control 53, 274-283.

WANG, S. \& TAN, W. 2009 The onset of Darcy Brinkman thermo solutal convection in a horizontal porous media. Phys. Lett. A 373 (7), 776-780.

Weir, G.J., White, S.P. \& Kissling, W.M. 1995 Reservoir storage and containment of greenhouse gases. Energy Convers. Manage. 36 (6-9), 531-534.

Weir, G.J., White, S.P. \& Kissling, W.M. 1996 Reservoir storage and containment of greenhouse gases. Trans. Porous Med. 23 (1), 37-60.

Wen, B., Ahkbari, D., Zhang, L. \& Hesse, M.A. 2018 a Dynamics of convective carbon dioxide dissolution in a closed porous media system. Preprint. arXiv:1801.02537.

Wen, B., Chang, K.W. \& Hesse, M.A. $2018 b$ Convection in porous media with dispersion. Phys. Rev. Fluids 3, 123801.

WEN, B., CoRson, L.T. \& ChINI, G.P. 2015 Structure and stability of steady porous medium convection at large Rayleigh number. J. Fluid Mech. 772, 197-224.

WhitAKER, S. 1969 Advances in theory of fluid motion in porous media. Ind. Engng Chem. 61 (12), 14-28.

Whitaker, S. 1986 Flow in porous media I: a theoretical derivation of Darcy's law. Trans. Porous Med. 1 (1), 3-25.

YANG, K. \& VAFAI, K. 2011 Analysis of heat flux bifurcation inside porous media incorporating inertial and dispersion effects-an exact solution. Intl J. Heat Mass Transfer 54 (25), 5286-5297.

ZARipov, S.K., Mardanov, R.F. \& SharafutdinOV, V.F. 2019 Determination of Brinkman model parameters using stokes flow model. Trans. Porous Med. 130 (2), 529-557.

Zech, A., Attinger, S., Bellin, A., Cvetkovic, V., Dietrich, P., Fiori, A., Teutsch, G. \& Dagan, G. 2019 A critical analysis of transverse dispersivity field data. Groundwater 57 (4), 632-639.

ZhaO, M., WANG, S., Li, S.C., Zhang, Q.Y. \& MAHABALESHWAR, U.S. 2018 Chaotic Darcy- Brinkman convection in a fluid saturated porous layer subjected to gravity modulation. Results Phys. 9, 1468-1480. 